\title{
Geochemical and Mineralogical Characteristics of the Middle Jurassic Coals from the Tongjialiang Mine in the Northern Datong Coalfield, Shanxi Province, China
}

\author{
Yue Yuan ${ }^{1,2,3}$, Shuheng Tang ${ }^{1,2,3, *}$ and Songhang Zhang ${ }^{1,2,3}$ \\ 1 School of Energy Resources, China University of Geosciences (Beijing), Beijing 100083, China; \\ yuanyue@cugb.edu.cn (Y.Y.); zhangsh@cugb.edu.cn (S.Z.) \\ 2 Beijing Key Laboratory of Unconventional Natural Gas Geological Evaluation and Development \\ Engineering, China University of Geosciences, Beijing 100083, China \\ 3 Coal Reservoir Laboratory of National Engineering Research Center of CBM Development \& Utilization, \\ China University of Geosciences, Beijing 100083, China \\ * Correspondence: tangsh@cugb.edu.cn; Tel.: +86-10-8232-2005
}

Received: 24 January 2019; Accepted: 12 March 2019; Published: 16 March 2019

\begin{abstract}
There is limited information available on the minerals and elements present in the Jurassic coals from Datong Coalfield. This paper investigates the geochemical and mineralogical characteristics of the Middle Jurassic coals from the Tongjialiang Mine using X-ray powder diffraction (XRD), X-ray fluorescence spectrometry (XRF), inductively coupled plasma mass spectrometry (ICP-MS), and scanning electron microscopy in combination with energy-dispersive X-ray spectrometry (SEM-EDS). No.12 coal is a low-medium volatile bituminous coal and is characterized by low ash yield content, low moisture content, and ultra-low sulfur content. Compared with Chinese coals, the Tongiialiang coals have slightly higher average percentages of $\mathrm{MgO}$ and $\mathrm{P}_{2} \mathrm{O}_{5}$, and lower average percentages of the other major oxides, including $\mathrm{SiO}_{2}, \mathrm{TiO}_{2}, \mathrm{Al}_{2} \mathrm{O}_{3}, \mathrm{Fe}_{2} \mathrm{O}_{3}, \mathrm{CaO}$, $\mathrm{MnO}, \mathrm{Na}_{2} \mathrm{O}$, and $\mathrm{K}_{2} \mathrm{O}$. Compared with the World hard coals, $\mathrm{Be}, \mathrm{Cr}, \mathrm{Co}, \mathrm{Ni}, \mathrm{Ge}, \mathrm{Sn}$, Ta, and $\mathrm{W}$ are slightly enriched in the Tongjialiang coals. The concentrations of $\mathrm{Li}, \mathrm{F}, \mathrm{Sc}, \mathrm{V}, \mathrm{Cu}, \mathrm{Ga}, \mathrm{Se}, \mathrm{Sr}, \mathrm{Zr}, \mathrm{Nb}, \mathrm{Hf}$, $\mathrm{Pb}$, Th, and $\mathrm{U}$ are close to the average values of the world's hard coals. The minerals in No.12 coal mainly include quartz, kaolinite, siderite, and ankerite, along with smaller amounts of pyrite, illite, calcite, and rutile. The formation of syngenetic siderite in No.12 coal is related to the weathering of biotite in the gneiss of the Yinshan Upland. The modes of occurrence of ankerite indicate that the coals may be affected by the injection of low temperature hydrothermal fluids. It is noteworthy that a portion of epigenetic ankerite may be a product of metasomatism between syngenetic siderite and the epigenetic Fe-Mg-Ca rich hydrothermal fluids. The ratios of $\mathrm{Al}_{2} \mathrm{O}_{3} / \mathrm{TiO}_{2}$, $\mathrm{REY}$ (rare earth elements and yittrium) enrichment patterns, the modes of occurrence of siderite and ankerite, as well as the enriched lithophile and siderophile elements indicate that the No.12 coal may have originated from the Yinshan Upland and may also have been influenced by low temperature hydrothermal fluids that might have circulated in the coal basin.
\end{abstract}

Keywords: major elements; trace elements; minerals; Jurassic coals; Datong coalfield

\section{Introduction}

In China, coal reserves are abundant, and coal is considered to be the most important mining resource. Under some specific geological conditions, coals may be enriched in some metal elements [1-3]. Understanding the concentrations, modes of occurrence, distributions, and origins of 
minerals and rare metal elements in coal would not only provide further evidence of the formation mechanisms for these rare metal elements, but also enhance the economic value of the coals that are enriched in rare metal components [4-7]. Moreover, the study of enrichment mechanisms of toxic elements in coals may be of great significance to reduce the adverse effects on human health and environment due to the release of toxic elements during the coal utilization [8-10]. Generally, minerals—as the main carrier of elements-are the most important inorganic components in coals. In some cases, elements such as Ge and $\mathrm{U}$ are bound in organic matters [11-14]. Additionally, it has been suggested that the minerals in coals might be used as indicators for the sediment source region, depositional conditions of the original peat swamp, geological history of coal-bearing sequences and the regional tectonics $[15,16]$. From the perspective of utilization, the minerals in coal would directly affect the calorific value of coal as well as the processing and utilization of coal. Improper combustion of coal and release of toxic elements present within it are also the main causes of pollution, corrosion and wear during coking [17]. The rational utilization of mineral resources plays an important role on the development of the economy and society $[18,19]$.

The Datong Coalfield is one of the largest coal producers of China and is located in northern Shanxi Province, which is adjacent to Shaanxi Province, Hebei Province, and Inner Mongolia Autonomous Region (Figure 1). The Datong coal-bearing basin, consisting of the Late Paleozoic Permian-Carboniferous and the Mesozoic Jurassic coal-bearing strata, has attracted much attention for its special characteristics, such as basin evolution, sedimentation, coal formation, and metamorphism [20-22]. Some studies have shown that the late Paleozoic coals from Datong Coalfield are associated with high concentrations of some potentially valuable and toxic elements. For example, $\mathrm{Li}, \mathrm{F}, \mathrm{Ga}, \mathrm{Tl}, \mathrm{Hg}$, and REY (rare earth elements and yitrrium) are slightly enriched in the No.4 coal from the Yongdingzhuang Mine [23]. Wang et al. reported that $\mathrm{Ga}$ and $\mathrm{U}$ are relatively enriched in the Permian-Carboniferous coals from the northern Datong Coalfield [24]. Another study reported that $\mathrm{Mn}, \mathrm{As}$, and Se have slightly higher concentration in the coals from Baidong Mine and Qifengshan Mine, which can have adverse effects on human health and environment during utilization [25]. Several potentially valuable elements, such as $\mathrm{Al}, \mathrm{Li}, \mathrm{Ge}, \mathrm{Zr}$, and $\mathrm{Hf}$, and potentially toxic elements, including $\mathrm{Be}, \mathrm{As}, \mathrm{Cd}$, and $\mathrm{Pb}$, are slightly enriched in the No.5 coal from Yanzishan Mine (data not published yet).

In the Early and Middle Jurassic, the coal-bearing rock series which were deposited under the fluvial and lacustrine sedimentary environment were superimposed on the Late Paleozoic strata [20-22]. Thus, the extremely thick coal seams of the Datong Formation were formed, which are of significant industrial value today [20-22]. Previous investigations on geochemical and mineralogical characteristics of the Jurassic coals from the Datong Coalfield are very scarce.

The present paper investigates the mineralogical and geochemical characteristics of the Jurassic coals from the Datong Coalfield, in order to evaluate different geological factors that may have controlled the abundance and distribution of trace elements and minerals in the coals. 


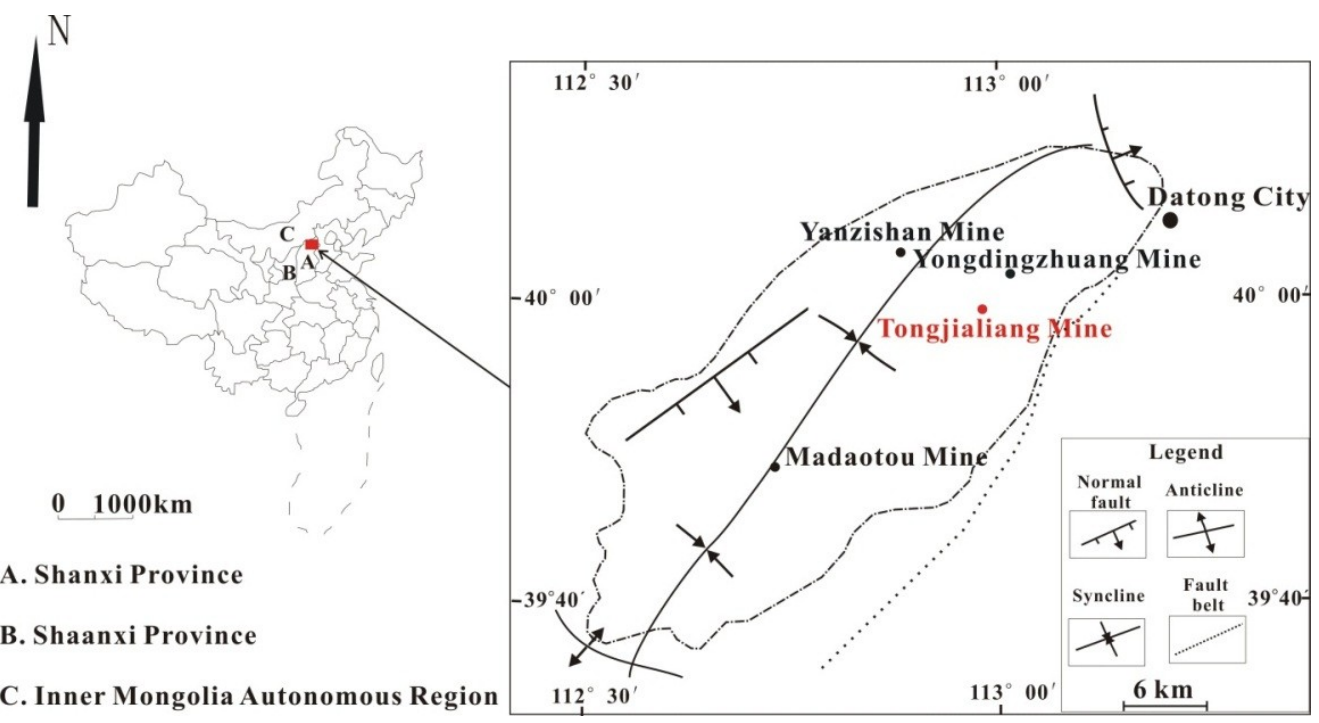

Figure 1. The location and geological map of the Datong Coalfield.

\section{Geological Setting}

The Datong Coalfield is located to the south of the Yinshan Upland and stretches $50 \mathrm{~km}$ long (NE-SW) and $30 \mathrm{~km}$ wide (NW-SE), covering a total area of $1900 \mathrm{~km}^{2}$ [20-22,26,27]. It is bound by the Pingwang-Emaokou fault to the west, by the Lvliang mountain syncline to the east, and by the Hongtao mountain syncline to the north $[20-22,26,27]$. The orogenic movement that occurred in the Early Paleozoic was defined by the Caledonian tectonic movement, which resulted in the uplift of the Datong coal-bearing basin [20-22,26,27]. The Datong basin with the Upper Carboniferous-Permian and Jurassic coal-bearing strata was formed after the Caledonian orogeny in the region [20-22,26,27]. The Indosinian movements caused the coalfield uplift, resulting in weathering and erosion of the Triassic strata. The magmatic activities consisted of the Indosinian lamprophyre and Yanshanian diabase invasions in the coalfield area [20-22,26,27]. A series of reverse faults was developed on the southern and southeastern margins of the basin including from north to south in the Qingciyao Fault, Wangjiayuan Fault, Meiyukou Fault, and Emaokou Fault. These faults probably provided channels for hydrothermal injections into the coal-bearing deposits [20-22,26,27]

The sedimentary sequence in the Jurassic coal-bearing strata of the Datong Coalfield mainly includes the Lower Jurassic Yongdingzhuang Formation, Middle Jurassic Datong Formation, and Middle Jurassic Yungang Formation [20-22]. The Datong Formation, the major coal-bearing unit in this area, contains several types of rocks including the conglomerate, gritstone, sandstone, medium sandstone, fine sandstone, and siltstone (Figure 2) [20-22]. In addition, it contains eight mineable coal seams, identified as Nos. 2, 3, 7, 10, 11, 12, 14, and 15. The coal-bearing sequence of the Datong Formation has the total thickness of $235 \mathrm{~m}$ (Figure 2) and is dominated by the fluvial and lacustrine sedimentary environment [20-22]. The No.12 coal seam is stable and widely distributed throughout the Datong Coalfield. The thickness of No.12 coal seam ranges from 0.35 to $7.90 \mathrm{~m}$, with an average of $2.80 \mathrm{~m}$ [20-22]. The roof and floor samples in No.12 coal seam are characterized by fine sandstone and siltstone, respectively (Figure 2) [20-22]. 


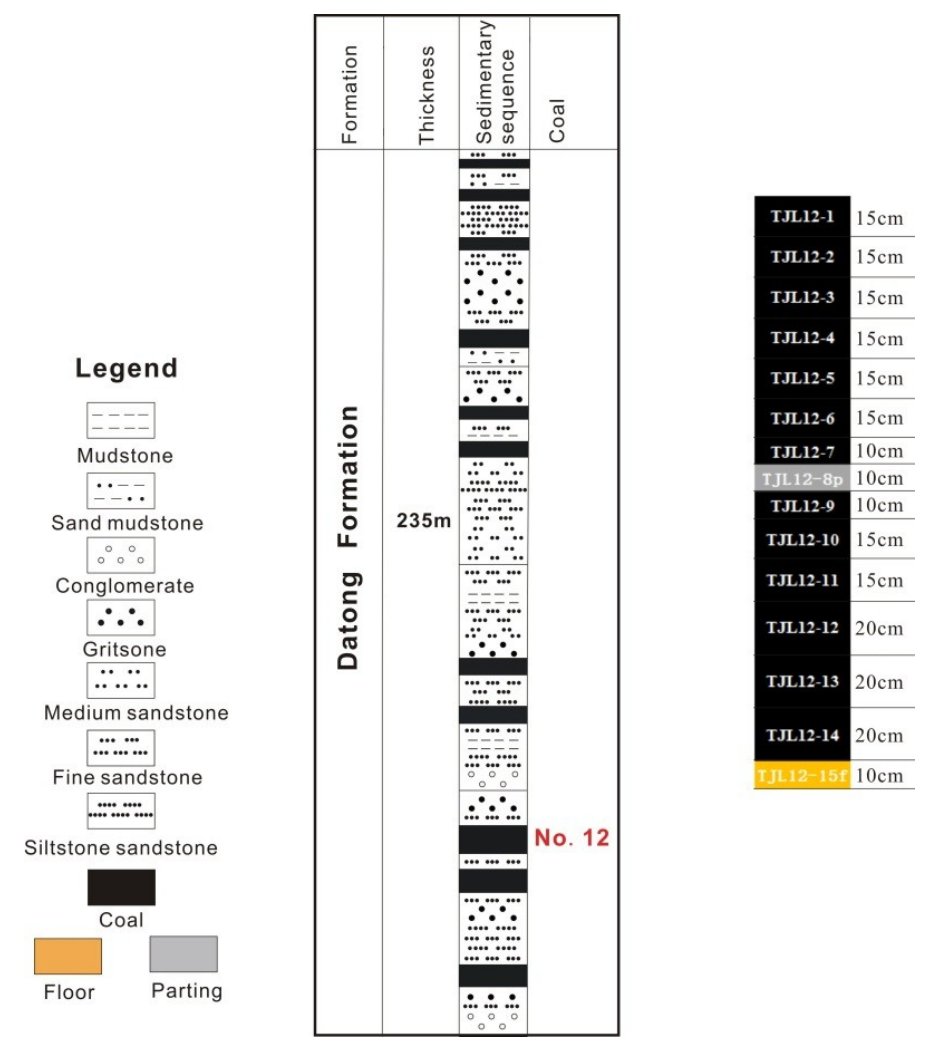

Figure 2. General stratigraphic sequence of the Datong Formation from the Datong Coalfield.

\section{Samples and Analytical Procedure}

The collected samples were placed in a grinder whose material is hadifield steel for pulverization, and the pulverized samples were screened by 18-40 mesh and 200 mesh sieves, respectively. The screened samples were immediately placed in a plastic bag and sealed. After a sample was ground, the grinder and sieve were washed with distilled water and then dried, in order to avoid contaminating other samples. The sample crushing was performed at the Beijing Key Laboratory of Unconventional Natural Gas Geological Evaluation and Development Engineering of China University of Geosciences (Beijing).

Proximate analyses of the coals, including moisture, ash yield, volatile matter, total sulfur, and forms of sulfur were performed based on ASTM (American Society for Testing and Materials) Standards D3173-11, D3174-11, D3175-11, D3177-02, and D2492-02, respectively [28-32].

The mineralogical composition was determined by microscopy (Leica DM 2500P microscope by Leica Microsystems, Solms, Germany). Low-temperature ashing of coal was performed on an EMITECH K1050 plasma asher (Quorum, Ashford, UK), with the temperature maintained at less than $150{ }^{\circ} \mathrm{C}$. X-ray diffraction (XRD) analyses of the low-temperature ashes were performed with $\mathrm{Ni}$-filtered $\mathrm{Cu}-\mathrm{K} \alpha$ radiation and a scintillation detector. The XRD patterns were recorded over a $2 \theta$ interval from $2.5-70^{\circ}$, with a step size of $0.01^{\circ}$. In order to determine the mineral composition from the X-ray diffractograms, the Siroquant ${ }^{\mathrm{TM}}$ commercial interpretation software developed by Taylor was used [33]. Further details of this technique have been provided by Ward and Ruan and Ward [34-36].

Coal samples of 80-mesh particle size were used for analysis of the mineralogical composition. A scanning electron microscope (HITACHI UHR FE-SEM, SU8220, HITACHI, Tokyo, Japan) equipped with an energy-dispersive X-ray spectrometer (SEM-EDS) was used to study the mineral distribution as well as the distribution patterns of several elements of interest in the coal.

Portions of all 15 samples were crushed into 200 mesh prior to elemental analysis. The 200 mesh samples were heated to $815^{\circ} \mathrm{C}$ to create high-temperature ashes, which were then formed into tablets and measured via X-ray fluorescence spectrometry (XRF) (Thermo ADVANT XP+, Thermo Fisher 
Scientific, Waltham, MA, USA). All the residues were used to determine the contents of major element oxides $\left(\mathrm{SiO}_{2}, \mathrm{TiO}_{2}, \mathrm{Al}_{2} \mathrm{O}_{3}, \mathrm{Fe}_{2} \mathrm{O}_{3}, \mathrm{MgO}, \mathrm{CaO}, \mathrm{MnO}, \mathrm{Na}_{2} \mathrm{O}, \mathrm{K}_{2} \mathrm{O}\right.$, and $\left.\mathrm{P}_{2} \mathrm{O}_{5}\right)$. Trace elements, including $\mathrm{REY}$ (rare earth elements and yittrium), except for $\mathrm{As}, \mathrm{Se}, \mathrm{Hg}$, and $\mathrm{F}$, were determined via inductively coupled plasma mass spectrometry (ICP-MS) [37]. For ICP-MS, 200-mesh samples were digested using an UltraClave Microwave High Pressure Reactor (Milestone, Sorisole, Italy). The basic load for the digestion tank was composed of $330 \mathrm{ml}$ distilled $\mathrm{H}_{2} \mathrm{O}, 30 \mathrm{ml} 30 \% \mathrm{H}_{2} \mathrm{O}_{2}$, and $2 \mathrm{ml} 98 \% \mathrm{H}_{2} \mathrm{SO}_{4}$. The initial nitrogen pressure was set to 50 bar, the highest temperature was set to $240{ }^{\circ} \mathrm{C}$, and the digestion process lasted for $75 \mathrm{~min}$. The reagents for the $50 \mathrm{mg}$ sample digestion were $5 \mathrm{ml} \mathrm{40 \%} \mathrm{HF}$, $2 \mathrm{ml} \mathrm{65 \%} \mathrm{HNO}_{3}$, and $1 \mathrm{ml} \mathrm{30 \%} \mathrm{H}_{2} \mathrm{O}_{2}$. Multi-element standards (Inorganic Ventures: CCS-1, CCS-4, CCS-5, and CCS-6) were used for the calibration of trace element concentrations [37]. Arsenic and Se were analyzed via ICP-MS with collision/reaction cell technology (ICP-CCT-MS) [38]. Fluorine was determined via pyrohydrolysis combined with an ion selective electrode (ISE) based on the ASTM method [39]. Mercury was determined using a Milestone DMA-80 Hg analyzer (with a 0.005-ng detection limit) (Milestone, Sorisole, Italy) [40].

The quantitative analyses of minerals and determinations of elements were completed at the State Key Laboratory of Coal Resources and Safe Mining of China University of Mining and Technology (Beijing, China).

\section{Results}

\subsection{Proximate Analysis}

Table 1 summarizes the results of total sulfur content and proximate analysis for the No.12 coal from the Tongjialiang mine in Datong Coalfield. According to the Chinese National Standards GB/T15224.1 [41] and 2 [42], MT/T 849 [43] and MT/T 850 [44], the No.12 coal is characterized by low ash (3.63-34.29\%, mean 9.79\%), low moisture (1.3-2.58\%, mean 2.23\%), medium volatile (14.1-39.67\%, mean $20.98 \%)$ and ultra-low sulfur $(0.13-0.33 \%$, mean $0.25 \%)$ coals. The content of volatile matter in No.12 coal indicates that it is a low-medium volatile bituminous coal based on ASTM Standard D388-15, 2015 [45]. The total sulfur consists of inorganic sulfur (including pyritic sulfur and sulfate sulfur) and organic sulfur, and pyritic sulfur is a dominant form in all the samples. The percentage of total sulfur is low $(<1 \%)$, which indicates that total sulfur is mainly derived from coal-forming plants [46].

Table 1. Bench thickness $(\mathrm{cm})$, proximate, and forms of sulfur (\%) of No.12 coal samples from the Tongjialiang Mine.

\begin{tabular}{ccccccccc}
\hline Sample & Thickness & $\mathbf{M}_{\mathbf{a d}}$ & $\mathbf{A}_{\mathbf{d}}$ & $\mathbf{V}_{\mathbf{d a f}}$ & $\mathbf{S}_{\text {t.d }}$ & $\mathbf{S}_{\mathbf{p . d}}$ & $\mathbf{S}_{\text {s.d }}$ & $\mathbf{S}_{\mathbf{o . d}}$ \\
\hline TJL12-1 & 15 & 2.22 & 4.1 & 22.75 & 0.33 & 0.19 & 0.01 & 0.13 \\
TJL12-2 & 15 & 2.23 & 14.38 & 23.09 & 0.33 & 0.28 & 0.01 & 0.04 \\
TJL12-3 & 15 & 2.12 & 6.79 & 18.58 & 0.25 & 0.24 & 0.01 & 0.00 \\
TJL12-4 & 15 & 2.58 & 4.78 & 19.78 & 0.25 & 0.22 & 0.01 & 0.02 \\
TJL12-5 & 15 & 2.16 & 5.08 & 15.73 & 0.31 & 0.28 & 0.01 & 0.02 \\
TJL12-6 & 15 & 2.51 & 3.63 & 20.97 & 0.29 & 0.25 & 0.01 & 0.03 \\
TJL12-7 & 10 & 2.58 & 4.76 & 25.26 & 0.25 & 0.18 & 0.01 & 0.06 \\
TJL12-8p & 10 & 0.77 & 85.36 & 73.09 & 0.08 & 0.08 & 0.00 & 0.00 \\
TJL12-9 & 10 & 2.2 & 15.71 & 14.1 & 0.19 & 0.14 & 0.00 & 0.05 \\
TJL12-10 & 15 & 2.34 & 7.15 & 16.29 & 0.21 & 0.16 & 0.01 & 0.04 \\
TJL12-11 & 15 & 2.47 & 4.17 & 23.65 & 0.27 & 0.13 & 0.01 & 0.13 \\
TJL12-12 & 20 & 2.17 & 11.81 & 16.35 & 0.29 & 0.14 & 0.01 & 0.14 \\
TJL12-13 & 20 & 2.1 & 10.56 & 16.57 & 0.19 & 0.14 & 0.01 & 0.04 \\
TJL12-14 & 20 & 1.3 & 34.29 & 39.67 & 0.13 & 0.12 & 0.01 & 0.00 \\
TJL12-15f & 10 & 0.73 & 88.9 & 78.58 & 0.05 & 0.04 & 0.01 & 0.00 \\
AVE & - & 2.23 & 9.79 & 20.98 & 0.25 & 0.19 & 0.01 & 0.05 \\
\hline
\end{tabular}

$\mathrm{M}$, moisture; A, ash yield; V, volatile matter; ad, air-dry basis; d, dry basis; daf, dry and ash-free basis; $\mathrm{S}_{\mathrm{t}}$, total sulfur; $S_{p}$, pyritic sulfur; $S_{s}$, sulfate sulfur; $S_{o}$, organic sulfur. 


\subsection{Geochemistry}

\subsubsection{Major Elements}

As shown in Table 2, the Tongjialiang coals have slightly higher average percentages of $\mathrm{MgO}$ (mean $0.38 \%$ ) and $\mathrm{P}_{2} \mathrm{O}_{5}$ (mean $0.11 \%$ ), and lower average percentages of the other major oxides, including $\mathrm{SiO}_{2}$ (mean 5.44\%), $\mathrm{TiO}_{2}$ (mean 0.04\%), $\mathrm{Al}_{2} \mathrm{O}_{3}$ (mean 1.54\%), $\mathrm{Fe}_{2} \mathrm{O}_{3}$ (mean 1.28\%), $\mathrm{CaO}$ (mean $0.64 \%$ ), $\mathrm{MnO}$ (mean $0.01 \%$ ), $\mathrm{Na}_{2} \mathrm{O}$ (mean $0.03 \%$ ), and $\mathrm{K}_{2} \mathrm{O}$ (mean $0.05 \%$ ), compared with Chinese coals reported by Dai et al. [3]. Due to the low ash yield, the Tongjialiang coals have relatively low average percentages of all the major oxides when converted to a whole-coal basis. $\mathrm{SiO}_{2}$ and $\mathrm{Al}_{2} \mathrm{O}_{3}$ are the major oxides in all the samples, although high proportions of $\mathrm{Fe}_{2} \mathrm{O}_{3}$ occur in samples TJL12-13 and TJL12-14.

$\mathrm{SiO}_{2} / \mathrm{Al}_{2} \mathrm{O}_{3}$ ratios for the samples (mean 4.06) are distinctly higher than the theoretical ratios for kaolinite (1.18) and Chinese coals (1.42). Therefore, a large amount of quartz is likely present in the mineral matter. Aluminum and Ti are regarded as essentially immobile elements due to the low solubility of their oxides and hydroxides in low temperature aqueous solutions [47]. Therefore, the ratios of $\mathrm{Al}_{2} \mathrm{O}_{3}$ and $\mathrm{TiO}_{2}$ should be close to the characteristics of their source rocks $[47,48]$. $\mathrm{Al}_{2} \mathrm{O}_{3} / \mathrm{TiO}_{2}$ ratios for the coal bench samples range from 20.63 to 86.62 with an average of 41.20.

Table 2. Concentrations of major oxides in Tongjialiang coal (\%) (on a whole-coal basis).

\begin{tabular}{cccccccccccccc}
\hline Sample & $\mathrm{LOI}$ & $\mathrm{SiO}_{2}$ & $\mathrm{TiO}_{2}$ & $\mathrm{Al}_{2} \mathbf{O}_{3}$ & $\mathrm{Fe}_{2} \mathbf{O}_{3}$ & $\mathbf{M g O}$ & $\mathrm{CaO}$ & $\mathbf{M n O}$ & $\mathbf{N a}_{2} \mathbf{O}$ & $\mathbf{K}_{2} \mathbf{O}$ & $\mathbf{P}_{2} \mathbf{O}_{5}$ & $\mathbf{S i O}_{2} / \mathrm{Al}_{2} \mathbf{O}_{3}$ & $\mathbf{A l}_{2} \mathbf{O}_{3} / \mathrm{TiO}_{2}$ \\
\hline TJL12-1 & 95.9 & 3.20 & 0.02 & 0.48 & 0.11 & 0.03 & 0.08 & 0.001 & 0.02 & 0.02 & 0.05 & 6.63 & 20.36 \\
TJL12-2 & 85.62 & 11.60 & 0.04 & 2.35 & 0.13 & 0.05 & 0.03 & 0.001 & 0.02 & 0.11 & 0.01 & 4.93 & 56.19 \\
TJL12-3 & 93.21 & 5.36 & 0.03 & 1.13 & 0.08 & 0.05 & 0.07 & 0.001 & 0.02 & 0.05 & 0.04 & 4.74 & 44.49 \\
TJL12-4 & 95.22 & 3.60 & 0.03 & 0.68 & 0.07 & 0.10 & 0.09 & $\mathrm{bdl}$ & 0.07 & 0.03 & 0.06 & 5.26 & 23.46 \\
TJL12-5 & 94.92 & 4.29 & 0.03 & 0.66 & 0.02 & 0.01 & 0.01 & 0.000 & 0.01 & 0.02 & 0.03 & 6.54 & 24.09 \\
TJL12-6 & 96.37 & 2.60 & 0.03 & 0.68 & 0.03 & 0.02 & 0.12 & 0.000 & 0.01 & 0.03 & 0.10 & 3.79 & 22.93 \\
TJL12-7 & 95.24 & 1.35 & 0.01 & 0.83 & 0.48 & 0.48 & 0.99 & 0.004 & 0.01 & 0.04 & 0.08 & 1.62 & 70.93 \\
TJL12-8p & 14.64 & 42.73 & 0.59 & 35.59 & 2.23 & 1.19 & 1.37 & 0.009 & 0.22 & 0.82 & 0.18 & 1.20 & 60.32 \\
TJL12-9 & 84.29 & 11.55 & 0.04 & 3.58 & 0.09 & 0.11 & 0.13 & 0.001 & 0.05 & 0.12 & 0.08 & 3.23 & 86.62 \\
TJL12-10 & 92.85 & 5.18 & 0.02 & 1.37 & 0.11 & 0.05 & 0.11 & 0.001 & 0.01 & 0.05 & 0.18 & 3.79 & 56.37 \\
TJL12-11 & 95.83 & 2.65 & 0.03 & 0.85 & 0.15 & 0.03 & 0.20 & 0.001 & 0.01 & 0.03 & 0.20 & 3.12 & 25.17 \\
TJL12-12 & 88.19 & 6.45 & 0.08 & 3.12 & 0.78 & 0.31 & 0.73 & 0.004 & 0.02 & 0.08 & 0.01 & 2.07 & 38.57 \\
TJL12-13 & 89.44 & 5.14 & 0.04 & 1.10 & 3.14 & 0.22 & 0.46 & 0.031 & 0.02 & 0.01 & 0.28 & 4.66 & 27.16 \\
TJL12-14 & 65.71 & 7.70 & 0.08 & 3.19 & 11.44 & 3.43 & 5.28 & 0.109 & 0.08 & 0.03 & 0.34 & 2.41 & 39.28 \\
TJL12-15f & 11.1 & 53.27 & 0.88 & 28.95 & 0.92 & 0.63 & 0.15 & 0.004 & 0.10 & 3.61 & 0.16 & 1.84 & 33.06 \\
AVE & 90.21 & 5.44 & 0.04 & 1.54 & 1.28 & 0.38 & 0.64 & 0.01 & 0.03 & 0.05 & 0.11 & 4.06 & 41.20 \\
Chinese & - & & & & & & & & & & \\
coal & & & & & & & & & & & & \\
\end{tabular}

bdl: below detetion limit.

\subsubsection{Trace Elements}

The concentrations of trace elements (on an ash basis) in Tongjialiang coals, parting, and floor samples are listed in Table 3, along with the concentration coefficients $(\mathrm{CC}=$ the ratio of average elemental concentrations (on an ash basis) in the investigated coal/the average concentrations in World hard coals (on an ash basis)) [49]. Based on the relative enrichment of elements, the CC value can be divided into six categories: unusually enriched (CC $>100)$, significantly enriched $(10<C C$ $<100)$, enriched $(5<C C<10)$, slightly enriched $(2<\mathrm{CC}<5)$, normal $(0.5<\mathrm{CC}<2)$, and depleted $(\mathrm{CC}<0.5)[49]$.

As shown in Figure 3, compared with the World hard coals reported by Ketris and Yudovich [50], Be $(48.47 \mu \mathrm{g} / \mathrm{g}), \mathrm{Cr}(235.23 \mu \mathrm{g} / \mathrm{g}), \mathrm{Co}(145.71 \mu \mathrm{g} / \mathrm{g}), \mathrm{Ni}(346.83 \mu \mathrm{g} / \mathrm{g}), \mathrm{Ge}(278.48 \mu \mathrm{g} / \mathrm{g}), \mathrm{Sn}(62.27 \mu \mathrm{g} / \mathrm{g})$, Ta $(13.22 \mu \mathrm{g} / \mathrm{g})$, and $\mathrm{W}(23.91 \mu \mathrm{g} / \mathrm{g})$ are slightly enriched in the Tongjialiang coals. The concentrations of Li $(101.15 \mu \mathrm{g} / \mathrm{g}), \mathrm{F}(851.45 \mu \mathrm{g} / \mathrm{g})$, Sc $(17.67 \mu \mathrm{g} / \mathrm{g}), \mathrm{V}(221.32 \mu \mathrm{g} / \mathrm{g}), \mathrm{Cu}(45.86 \mu \mathrm{g} / \mathrm{g}), \mathrm{Ga}(22.69 \mu \mathrm{g} / \mathrm{g})$, Se $(4.12 \mu \mathrm{g} / \mathrm{g}), \mathrm{Sr}(528.96 \mu \mathrm{g} / \mathrm{g}), \mathrm{Zr}(446.06 \mu \mathrm{g} / \mathrm{g}), \mathrm{Nb}(26.00 \mu \mathrm{g} / \mathrm{g}), \mathrm{Hf}(12.55 \mu \mathrm{g} / \mathrm{g}), \mathrm{Pb}(41.46 \mu \mathrm{g} / \mathrm{g}), \mathrm{Th}$ $(11.86 \mu \mathrm{g} / \mathrm{g})$, and $\mathrm{U}(7.66 \mu \mathrm{g} / \mathrm{g})$ are close to the average values of the world's hard coals. Additionally, all the remaining elements are depleted in the Tongjialiang coals. 
Table 3. Concentrations of trace elements in the No.12 coal from the Tongjialiang mine ( $\mu \mathrm{g} / \mathrm{g}$ ) (on an ash basis).

\begin{tabular}{|c|c|c|c|c|c|c|c|c|c|c|c|c|c|c|c|c|c|}
\hline Sample & $\mathbf{L i}$ & Be & F & Sc & V & $\mathrm{Cr}$ & Co & $\mathrm{Ni}$ & $\mathrm{Cu}$ & $\mathrm{Zn}$ & Ga & Ge & As & Se & $\mathbf{R b}$ & $\mathrm{Sr}$ & $\mathrm{Zr}$ \\
\hline TJL12-1 & 84.18 & 235.02 & 521.11 & 76.17 & 2585.85 & 1065.64 & 502.39 & 1030.38 & 98.87 & 657.33 & 91.96 & 551.46 & 5.90 & 4.20 & 22.55 & 257.47 & 1219.75 \\
\hline TJL12-2 & 105.86 & 41.66 & 454.57 & 14.92 & 40.70 & 137.07 & 164.64 & 192.81 & 28.71 & bdl & 23.19 & 20.88 & 2.76 & 3.66 & 16.75 & 146.20 & 229.11 \\
\hline TJL12-3 & 95.05 & 27.43 & 293.47 & 7.10 & 25.67 & 92.41 & 147.33 & 181.86 & 34.71 & 96.23 & 13.11 & 12.50 & 2.04 & 0.25 & 9.92 & 204.48 & 182.04 \\
\hline TJL12-4 & 41.42 & 56.10 & 316.04 & 4.78 & 28.17 & 641.20 & 405.62 & 681.03 & 67.58 & bdl & 18.11 & 39.95 & bdl & bdl & bdl & 385.29 & 139.32 \\
\hline TJL12-5 & 128.60 & 45.05 & 784.97 & 45.48 & 14.04 & 193.26 & 138.47 & 282.18 & 40.36 & bdl & 17.75 & 15.98 & bdl & 3.93 & 76.69 & 1169.27 & 197.72 \\
\hline TJL12-6 & 48.83 & 86.82 & 1261.28 & 9.29 & 36.07 & 275.59 & 246.34 & 599.02 & 111.51 & bdl & 24.47 & 48.33 & 3.65 & 7.12 & bdl & 631.79 & 126.69 \\
\hline TJL12-7 & 25.83 & 66.41 & 888.53 & 14.54 & 21.47 & 51.87 & 107.92 & 457.11 & 32.89 & bdl & 25.49 & 15.28 & bdl & 5.72 & 1.94 & 984.48 & 2336.15 \\
\hline TJL12-8p & 39.00 & 1.53 & 1058.77 & 1.77 & 11.24 & 12.46 & 1.21 & 18.85 & 11.49 & 19.31 & 35.10 & 4.74 & 0.82 & 0.36 & 9.99 & 136.12 & 5.11 \\
\hline TJL12-9 & 235.94 & 9.90 & 620.90 & 8.45 & 17.16 & 58.49 & 6.19 & 51.74 & 17.88 & bdl & 12.92 & bdl & 0.64 & 3.35 & 13.07 & 96.05 & bdl \\
\hline TJL12-10 & 97.99 & 23.38 & 1417.30 & 13.99 & 27.83 & 132.04 & 24.64 & 196.42 & 49.69 & bdl & 21.25 & 12.16 & 3.36 & 12.41 & 3.87 & 1218.38 & 396.74 \\
\hline TJL12-11 & 59.78 & 24.99 & 2392.36 & 14.33 & 31.94 & 160.44 & 73.25 & 499.59 & 59.88 & bdl & 16.50 & 22.39 & 9.20 & 3.56 & bdl & 906.16 & 285.07 \\
\hline TJL12-12 & 236.77 & 6.67 & 493.66 & 12.48 & 34.53 & 61.25 & 18.34 & 121.37 & 24.03 & bdl & 17.46 & 2.18 & 10.65 & 1.15 & 9.35 & 193.50 & 147.76 \\
\hline TJL12-13 & 93.18 & 5.03 & 1411.02 & 6.65 & 11.68 & 119.53 & 21.26 & 107.38 & 22.11 & bdl & 4.50 & 1.85 & 3.67 & 3.80 & bdl & 229.84 & 79.54 \\
\hline TJL12-14 & 61.57 & 1.63 & 213.60 & 1.57 & 2.09 & 69.26 & 37.90 & 107.84 & 7.99 & 81.89 & 8.23 & 4.30 & 0.25 & 0.30 & 11.62 & 453.61 & 12.87 \\
\hline TJL12-15f & 80.78 & 1.83 & 1272.03 & 5.78 & 163.06 & 210.96 & 17.61 & 62.60 & 75.31 & 134.98 & 38.44 & 2.08 & 1.03 & 1.25 & 87.41 & 122.59 & 163.99 \\
\hline AVE & 101.15 & 48.47 & 851.45 & 17.67 & 221.32 & 235.23 & 145.71 & 346.83 & 45.86 & 278.48 & 22.69 & 62.27 & 3.83 & 4.12 & 18.42 & 528.96 & 446.06 \\
\hline World $^{1}$ & 82 & 12 & 630 & 24 & 170 & 82 & 26 & 52 & 74 & 110 & 36 & 18 & 48 & 7.60 & 110 & 730 & 230 \\
\hline$C C^{2}$ & 1.23 & 4.04 & 1.35 & 0.74 & 1.30 & 2.87 & 5.60 & 6.67 & 0.62 & 2.53 & 0.63 & 3.46 & 0.08 & 0.54 & 0.17 & 0.72 & 1.94 \\
\hline Sample & $\mathrm{Nb}$ & Mo & $\mathrm{Cd}$ & In & Sn & $\mathrm{Sb}$ & Cs & Ba & Hf & Ta & $\mathbf{W}$ & $\mathrm{Hg}$ & $\mathrm{Tl}$ & $\mathbf{P b}$ & $\mathbf{B i}$ & Th & $\mathbf{U}$ \\
\hline TJL12-1 & 79.84 & bdl & 2.08 & 0.05 & 1.16 & 2.46 & 0.72 & 548.18 & 23.86 & $\mathrm{bdl}$ & $\mathrm{bdl}$ & 0.37 & bdl & 170.83 & bdl & 4.30 & 8.55 \\
\hline TJL12-2 & 17.00 & 2.57 & 0.22 & 0.08 & 4.17 & 0.28 & 1.46 & 306.04 & 6.80 & 2.99 & bdl & 0.16 & 3.12 & 37.31 & bdl & 12.98 & 6.08 \\
\hline TJL12-3 & 10.56 & 1.79 & 0.59 & 0.03 & 1.17 & bdl & 0.47 & 354.99 & 4.81 & $\mathrm{bdl}$ & bdl & 0.10 & 1.64 & 36.88 & bdl & 4.72 & 5.08 \\
\hline TJL12-4 & 8.51 & 3.02 & bdl & 0.04 & 1.34 & bdl & bdl & 2088.53 & 2.89 & bdl & bdl & 0.08 & bdl & 17.82 & bdl & 0.84 & 4.36 \\
\hline TJL12-5 & 5.13 & bdl & 0.32 & 0.04 & 1.50 & bdl & 3.63 & 3587.73 & 4.46 & bdl & bdl & 0.07 & bdl & 20.59 & bdl & 67.06 & 6.59 \\
\hline TJL12-6 & 10.45 & 23.81 & bdl & 0.05 & 5.28 & bdl & bdl & 1977.09 & 3.02 & bdl & bdl & 0.11 & bdl & 25.13 & bdl & 9.46 & 19.80 \\
\hline TJL12-7 & 125.97 & 21.95 & bdl & 0.08 & 205.68 & 3.59 & 0.34 & 3469.39 & 80.99 & 58.22 & 44.72 & 0.07 & bdl & 53.81 & bdl & 3.34 & 13.86 \\
\hline TJL12-8p & 0.23 & $\mathrm{bdl}$ & 0.23 & 0.07 & bdl & bdl & 1.77 & 262.38 & 0.10 & $\mathrm{bdl}$ & 0.71 & 0.14 & 0.16 & 52.94 & 1.28 & 30.25 & 5.71 \\
\hline TJL12-9 & bdl & bdl & 0.19 & bdl & bdl & bdl & 0.40 & 232.01 & bdl & bdl & bdl & 0.05 & 0.28 & 27.68 & bdl & 13.58 & 8.45 \\
\hline TJL12-10 & 27.75 & 4.43 & 0.50 & 0.03 & 0.87 & 0.81 & bdl & 4367.22 & 9.87 & bdl & 0.22 & 0.07 & bdl & 61.75 & bdl & 6.28 & 17.83 \\
\hline TJL12-11 & 12.21 & 4.20 & 0.24 & 0.10 & 1.16 & bdl & bdl & 3052.17 & 7.04 & bdl & 33 & 0.19 & 1.16 & 69.96 & bdl & 5.45 & 3.96 \\
\hline TJL12-12 & 8.08 & 0.86 & 0.41 & 0.03 & 2.91 & bdl & 0.47 & 263.17 & 4.54 & 3.56 & bdl & 0.07 & 0.83 & 5.52 & bdl & 18.12 & 3.08 \\
\hline TJL12-13 & 5.94 & 0.04 & 0.13 & bdl & 0.79 & bdl & bdl & 1660.81 & 1.97 & 0.08 & bdl & 0.04 & 0.72 & 0.74 & bdl & 7.67 & 1.64 \\
\hline TJL12-14 & 0.60 & 2.43 & 0.13 & 0.01 & 0.27 & bdl & 0.69 & 1008.96 & 0.32 & 1.24 & 17.69 & 0.02 & 0.03 & 10.92 & bdl & 0.31 & 0.28 \\
\hline TJL12-15f & 15.88 & 2.50 & 0.46 & 0.11 & 1.95 & 0.11 & 7.84 & 486.70 & 4.48 & 1.24 & 1.51 & 0.03 & 0.79 & 50.20 & 0.19 & 6.16 & 3.00 \\
\hline AVE & 26.00 & 6.51 & 0.37 & 0.04 & 18.86 & 1.78 & 0.82 & 1762.79 & 12.55 & 13.22 & 23.91 & 0.11 & 1.11 & 41.46 & bdl & 11.86 & 7.66 \\
\hline World $^{1}$ & 22 & 14 & 1.10 & 0.11 & 8 & 5 & 8 & 980 & 9 & 2 & 7.80 & 0.62 & 4.60 & 38 & 4.30 & 23 & 1.90 \\
\hline $\mathrm{CC}^{2}$ & 1.18 & 0.46 & 0.34 & 0.38 & 2.36 & 0.36 & 0.10 & 1.80 & 1.39 & 6.61 & 3.07 & 0.17 & 0.24 & 1.09 & bdl & 0.52 & 0.51 \\
\hline
\end{tabular}

${ }^{1}$ World's hard coals reported by Ketris and Yodvich [50]; ${ }^{2} \mathrm{CC}=$ average concentrantion of the trace elements in coals (on an ash basis) vs. world's hard coals (on an ash basis); bdl: below detection limit. 


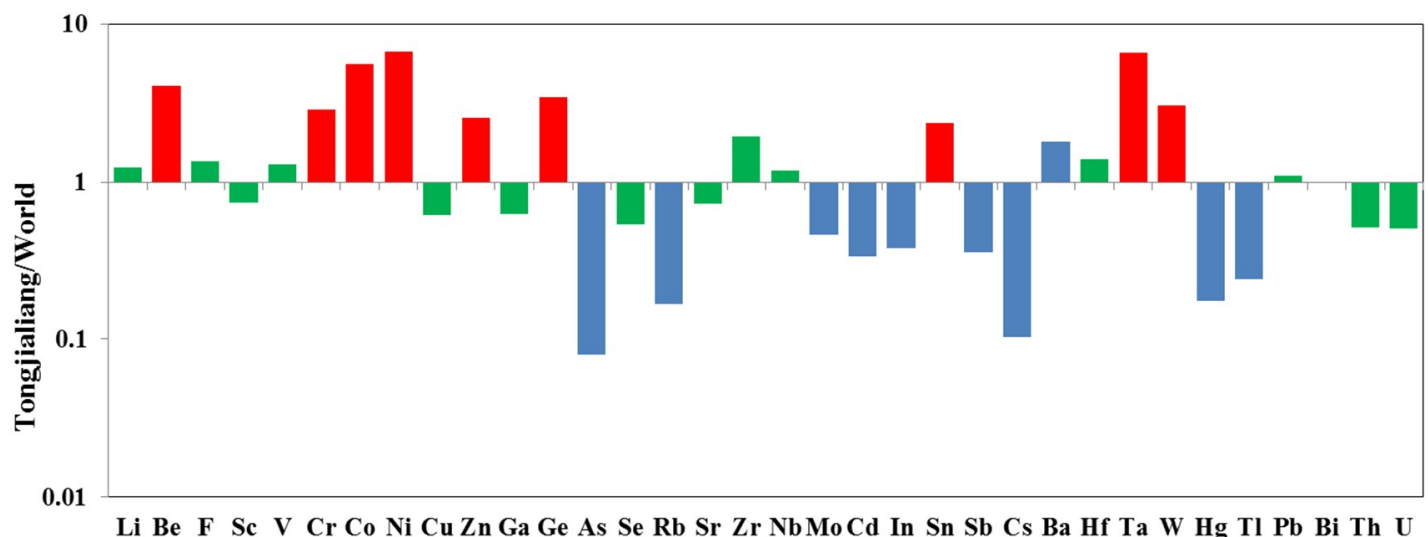

\section{$\mathrm{CC}<0.5$}

\section{$0.5<\mathrm{CC}<2$}

\section{$2<\mathrm{CC}<5$}

Figure 3. Concentration coefficients of trace elements in the No.12 coal, normalized by average concentrations in the world's hard coals.

\subsubsection{Rare Earth Elements}

In this study, a threefold geochemical classification of rare earth elements and yttrium (REY) was used. Based on Seredin-Dai's classification [51], the REYs are divided into three types: light (LREY including $\mathrm{La}, \mathrm{Ce}, \mathrm{Pr}, \mathrm{Nd}$, and $\mathrm{Sm}$ ), medium (MREY including, $\mathrm{Eu}, \mathrm{Gd}, \mathrm{Tb}, \mathrm{Dy}$, and $\mathrm{Y}$ ), and heavy (HREY including, $\mathrm{Ho}, \mathrm{Er}, \mathrm{Tm}, \mathrm{Yb}$, and $\mathrm{Lu}$ ). The concentrations and geochemical parameters of REY in No.12 coal are listed in Table 4. The concentration of REY in the coal ash samples ranges from $14.87 \mu \mathrm{g} / \mathrm{g}$ to $1172.47 \mu \mathrm{g} / \mathrm{g}$, with an average of $399.70 \mu \mathrm{g} / \mathrm{g}$, which is much lower than corresponding value for the world's hard coals $(445.3 \mu \mathrm{g} / \mathrm{g})$ [50]. The content of LREE $(9.09-1049.23 \mu \mathrm{g} / \mathrm{g}$, mean $303.75 \mu \mathrm{g} / \mathrm{g})$ is much higher than that of MREE $(4.94-199.03 \mu \mathrm{g} / \mathrm{g}$, mean $80.69 \mu \mathrm{g} / \mathrm{g})$ and HREE $(0.85-48.83 \mu \mathrm{g} / \mathrm{g}$, mean $15.26 \mu \mathrm{g} / \mathrm{g})$.

Compared with the Upper Continental Crust (UCC) [52], three types of coal benches are identified as: L-type (LREY; $\left.\mathrm{La}_{\mathrm{N}} / \mathrm{Lu}_{\mathrm{N}}>1\right)$, M-type (MREY; $\left.\mathrm{La}_{N} / \mathrm{Sm}_{\mathrm{N}}<1, \mathrm{Gd}_{\mathrm{N}} / \mathrm{Lu}_{\mathrm{N}}>1\right)$, and H-type (HREY; $\mathrm{La}_{N} / \mathrm{Lu}_{\mathrm{N}}<1$ ) [53]. The REY enrichment patterns of the Tongjialiang coals are shown in Figure 4. The samples of TJL12-4, TJL12-5 and TJL12-10, are characterized as L-type enrichment as well as one parting sample (TJL12-8p) and one floor sample (TJL12-15f). The H-type enrichment patterns include six coal bench samples, namely, TJL12-1, TJL12-2, TJL12-3, TJL12-9, TJL12-12, and TJL12-14. The samples of TJL12-6, TJL12-11, and TJL12-13 belong to the M-H type enrichment. Only sample TJL12-7 is characterized by L-M type enrichment.

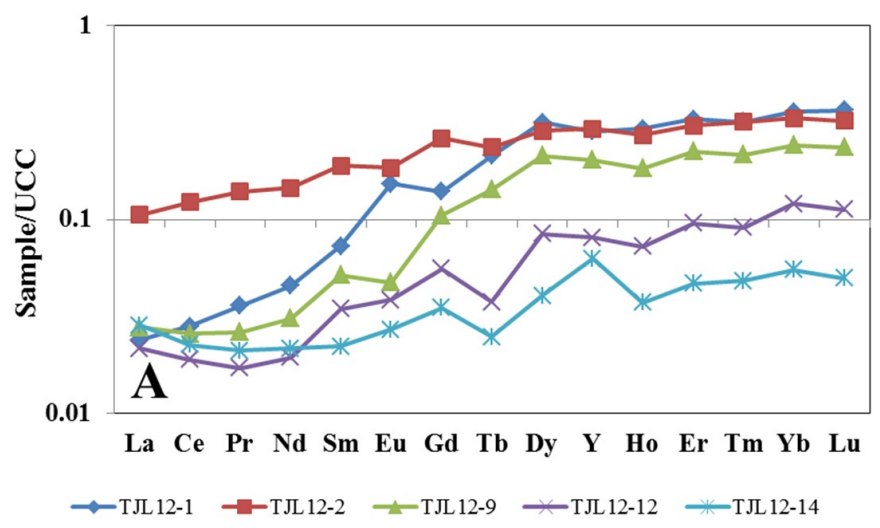

Figure 4. Cont. 

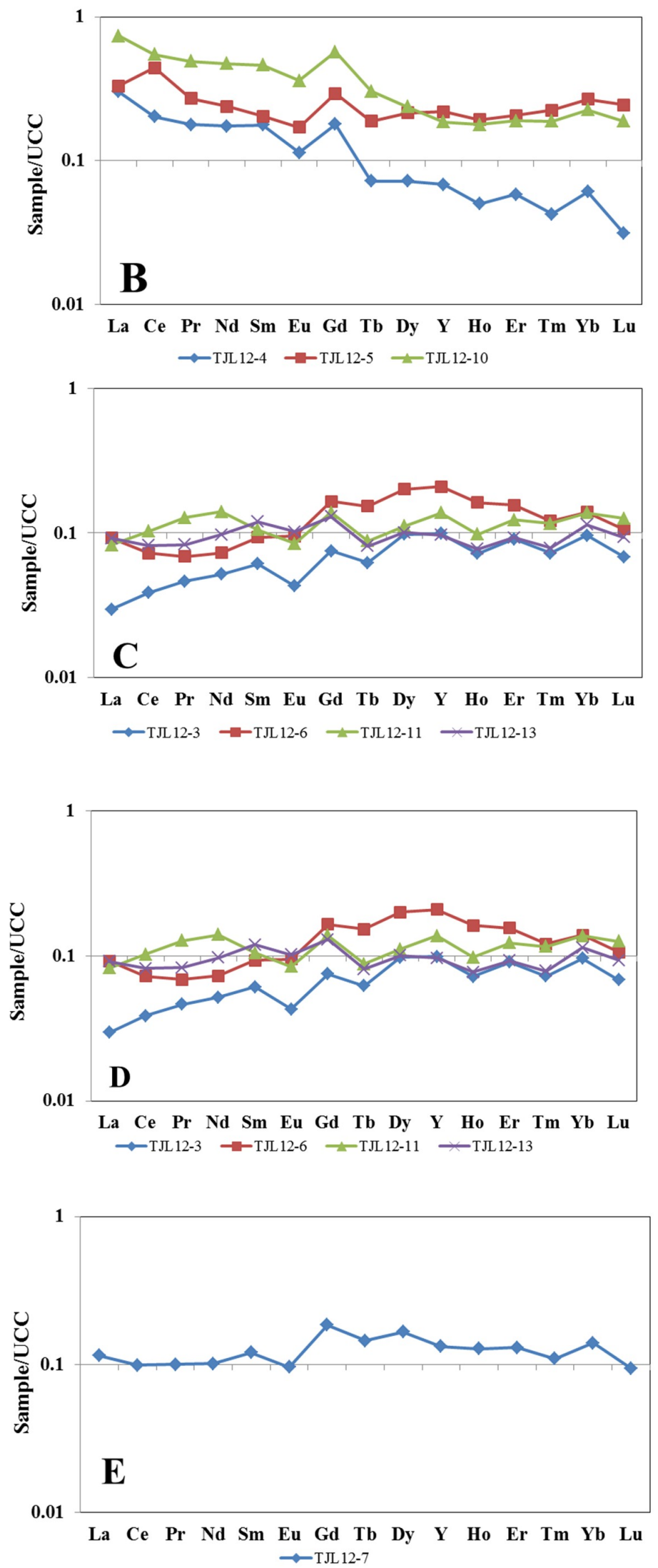

Figure 4. REY distribution patterns in the coal benches, roof, floor and partings, normalized to the upper continental crust (UCC) reported by Taylor and McLennan [52]; (A) pattern of H-type in coal benches; (B) pattern of L type in coal benches; (C) pattern of M-H type in coal benches; (D) pattern of L-M type in coal benches; (E) pattern of L-type in the floor and parting samples. 
Table 4. Concentration and geochemical parameters of REY in the No.12 coals ( $\mu \mathrm{g} / \mathrm{g}$ ) (on an ash basis).

\begin{tabular}{|c|c|c|c|c|c|c|c|c|c|c|c|c|c|c|c|c|}
\hline Elements & TJL12-1 & TJL12-2 & TJL12-3 & TJL12-4 & TJL12-5 & TJL12-6 & TJL12-7 & TJL12-8p & TJL12-9 & TJL12-10 & TJL12-11 & TJL12-12 & TJL12-13 & TJL12-14 & TJL12-15f & AVE \\
\hline $\mathrm{La}$ & 17.53 & 22.08 & 13.08 & 189.71 & 194.96 & 76.10 & 72.24 & 43.71 & 5.32 & 308.95 & 59.30 & 5.50 & 26.10 & 2.49 & 48.10 & 76.41 \\
\hline $\mathrm{Ce}$ & 43.90 & 54.75 & 36.47 & 270.42 & 559.35 & 127.57 & 133.24 & 65.20 & 10.50 & 489.90 & 157.25 & 10.25 & 49.79 & 4.23 & 105.87 & 149.82 \\
\hline $\operatorname{Pr}$ & 6.23 & 6.87 & 4.84 & 26.54 & 37.83 & 13.47 & 14.92 & 10.67 & 1.19 & 48.91 & 21.66 & 1.03 & 5.58 & 0.44 & 10.93 & 14.58 \\
\hline $\mathrm{Nd}$ & 28.88 & 26.41 & 19.80 & 94.54 & 122.09 & 52.18 & 55.33 & 35.99 & 5.10 & 172.34 & 87.29 & 4.28 & 24.01 & 1.64 & 39.63 & 53.38 \\
\hline $\mathrm{Sm}$ & 8.02 & 5.92 & 4.05 & 16.73 & 18.03 & 11.55 & 11.37 & 5.63 & 1.48 & 29.12 & 11.34 & 1.32 & 5.10 & 0.29 & 6.49 & 9.56 \\
\hline $\mathrm{Eu}$ & 3.28 & 1.13 & 0.56 & 2.10 & 2.96 & 2.31 & 1.78 & 0.65 & 0.27 & 4.43 & 1.79 & 0.29 & 0.85 & 0.07 & 1.43 & 1.68 \\
\hline $\mathrm{Gd}$ & 12.94 & 6.94 & 4.20 & 14.25 & 21.93 & 17.32 & 14.79 & 4.64 & 2.54 & 30.41 & 12.64 & 1.80 & 4.67 & 0.39 & 6.23 & 11.14 \\
\hline $\mathrm{Tb}$ & 3.33 & 1.05 & 0.59 & 0.96 & 2.37 & 2.69 & 1.94 & 0.43 & 0.58 & 2.72 & 1.35 & 0.20 & 0.49 & 0.05 & 0.75 & 1.41 \\
\hline Dy & 27.00 & 6.98 & 5.05 & 5.28 & 14.79 & 19.36 & 12.22 & 1.61 & 4.75 & 11.58 & 9.36 & 2.51 & 3.35 & 0.41 & 3.65 & 9.43 \\
\hline Y & 152.48 & 44.95 & 32.18 & 31.49 & 94.84 & 126.74 & 61.33 & 6.69 & 28.35 & 57.37 & 72.47 & 15.09 & 20.11 & 4.02 & 17.30 & 57.03 \\
\hline Ho & 5.75 & 1.52 & 0.85 & 0.84 & 3.04 & 3.57 & 2.16 & 0.29 & 0.93 & 1.99 & 1.88 & 0.49 & 0.59 & 0.09 & 0.69 & 1.82 \\
\hline Er & 18.45 & 4.87 & 3.08 & 2.81 & 9.35 & 9.90 & 6.30 & 0.82 & 3.28 & 6.08 & 6.80 & 1.86 & 2.02 & 0.31 & 1.86 & 5.78 \\
\hline $\mathrm{Tm}$ & 2.56 & 0.73 & 0.35 & 0.29 & 1.46 & 1.10 & 0.76 & 0.12 & 0.45 & 0.87 & 0.92 & 0.25 & 0.25 & 0.05 & 0.27 & 0.77 \\
\hline $\mathrm{Yb}$ & 19.22 & 5.11 & 3.11 & 2.81 & 11.60 & 8.47 & 6.47 & 0.87 & 3.38 & 6.95 & 7.29 & 2.25 & 2.38 & 0.35 & 1.63 & 6.11 \\
\hline $\mathrm{Lu}$ & 2.85 & 0.72 & 0.32 & 0.21 & 1.54 & 0.93 & 0.63 & 0.13 & 0.48 & 0.84 & 0.97 & 0.30 & 0.28 & 0.05 & 0.22 & 0.78 \\
\hline$\sum R E Y$ & 352.43 & 190.02 & 128.53 & 658.97 & 1096.13 & 473.27 & 395.48 & 177.46 & 68.60 & 1172.47 & 452.31 & 47.43 & 145.56 & 14.87 & 245.06 & 399.70 \\
\hline$\sum$ LREY & 104.56 & 116.03 & 78.24 & 597.93 & 932.26 & 280.87 & 287.10 & 161.20 & 23.58 & 1049.23 & 336.84 & 22.39 & 110.57 & 9.09 & 211.02 & 303.75 \\
\hline ¿MREY & 199.03 & 61.06 & 42.57 & 54.08 & 136.89 & 168.42 & 92.06 & 14.02 & 36.49 & 106.51 & 97.61 & 19.88 & 29.47 & 4.94 & 29.36 & 80.69 \\
\hline ¿HREY & 48.83 & 12.94 & 7.72 & 6.96 & 26.98 & 23.97 & 16.32 & 2.24 & 8.54 & 16.73 & 17.85 & 5.17 & 5.52 & 0.85 & 4.67 & 15.26 \\
\hline $\mathrm{La}_{N} / \mathrm{Lu}_{\mathrm{N}}$ & 0.07 & 0.33 & 0.43 & 9.65 & 1.35 & 0.87 & 1.22 & 3.56 & 0.12 & 3.92 & 0.66 & 0.19 & 0.98 & 0.57 & 2.29 & 1.57 \\
\hline $\mathrm{La}_{\mathrm{N}} / \mathrm{Sm}_{\mathrm{N}}$ & 0.33 & 0.56 & 0.48 & 1.70 & 1.62 & 0.99 & 0.95 & 1.17 & 0.54 & 1.59 & 0.78 & 0.63 & 0.77 & 1.28 & 1.11 & 0.94 \\
\hline $\mathrm{Gd}_{\mathrm{N}} / \mathrm{Lu}_{\mathrm{N}}$ & 0.39 & 0.79 & 0.78 & 2.94 & 1.09 & 1.18 & 1.32 & 3.08 & 0.43 & 2.53 & 1.00 & 0.46 & 1.13 & 0.64 & 2.22 & 1.13 \\
\hline$\delta \mathrm{Eu}$ & 1.28 & 0.90 & 0.70 & 0.80 & 0.86 & 0.84 & 0.75 & 0.69 & 0.58 & 0.88 & 0.85 & 1.09 & 0.95 & 1.18 & 1.20 & 0.90 \\
\hline Type & $\mathrm{H}$ & $\mathrm{H}$ & $\mathrm{H}$ & $\mathrm{L}$ & $\mathrm{L}$ & M-H & L-M & $\mathrm{L}$ & $\mathrm{H}$ & L & M-H & $\mathrm{H}$ & M-H & $\mathrm{H}$ & L & - \\
\hline
\end{tabular}

N. values normalized by the average REY content of Upper Continental Crust. $\delta \mathrm{Eu}=\mathrm{Eu}_{\mathrm{N}} / \mathrm{Eu}_{N^{*}}=\mathrm{Eu}_{\mathrm{N}} /\left(0.67 \times \mathrm{Sm}_{\mathrm{N}}+0.33 \times \mathrm{Tb}_{\mathrm{N}}\right)[53]$. 
Yan et al. reported that positive Eu anomalies could be caused by overestimation of Eu during ICP-MS analysis due to interference from $\mathrm{BaO}$ or $\mathrm{BaOH}$ [54]. If $\mathrm{Ba} / \mathrm{Eu}>1000$, the interfered Eu is highly elevated. Therefore, when samples contain Ba/Eu less than 1000, the interference of $\mathrm{Ba}$ on $\mathrm{Eu}$ can be ignored. Despite the positive correlation between Ba and Eu concentrations $(r=0.80)$, lower $\mathrm{Ba} / \mathrm{Eu}$ values (468.38 on an average) in the investigated samples indicate that the positive Eu anomalies in these samples are not caused by the interference of Ba. All the coal samples are characterized by weakly positive or no pronounced $\mathrm{Eu}$ anomalies $\left(\delta \mathrm{Eu}=\mathrm{Eu}_{\mathrm{N}} / \mathrm{Eu}_{\mathrm{N}}{ }^{*}=\mathrm{Eu}_{\mathrm{N}} /\left(0.67 \times \mathrm{Sm}_{\mathrm{N}}+0.33 \times\right.\right.$ $\mathrm{Tb}_{\mathrm{N}}$ ) ranging from $0.58-1.28$, with an average of 0.90$)$ [53].

\subsection{Abundances and Occurrence Modes of Minerals}

Table 5 lists the proportion of minerals in the coal LTAs (low temperature ashes), partings, roof, and floor samples identified by LTA-XRD and Siroquant. The mineralogical composition of the coal samples is mainly characterized by quartz, kaolinite, pyrite, siderite, and ankerite, while illite, I/S, calcite, rutile, anorthite, goyazite, and basanite are less abundant. Figure 5 shows the vertical variations of the contents of low temperature ashes and minerals in LTAs in the No.12 coal.

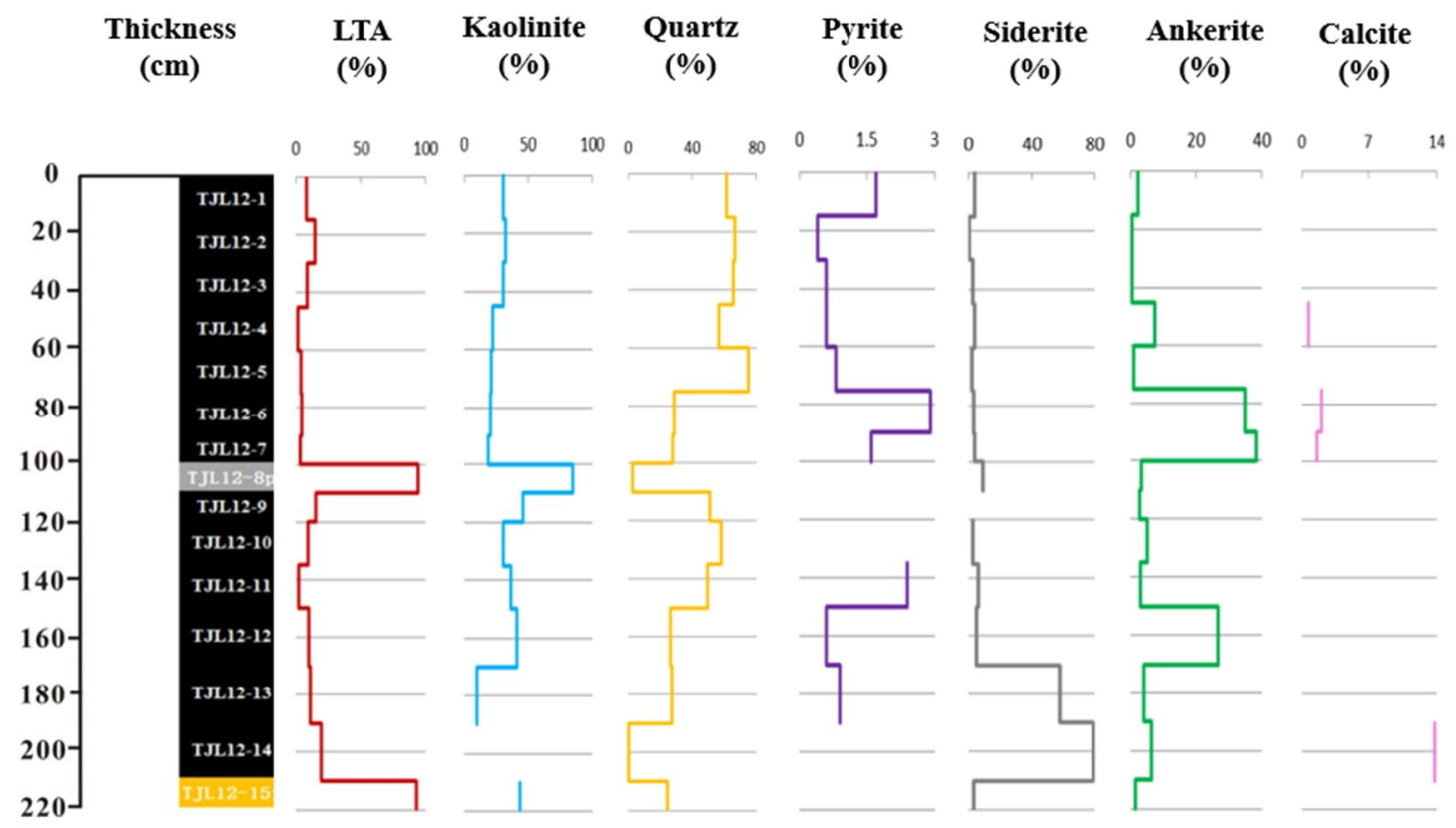

Figure 5. Vertical variations of low temperature ash yield and minerals in LTA and rock samples in the No.12 coal from the Tongjialiang mine. 
Table 5. Mineralogical compositions and contents of coal LTAs and non-coal samples in No.12 coal in the Tongjialiang Mine (\%) (on an ash basis).

\begin{tabular}{|c|c|c|c|c|c|c|c|c|c|c|c|c|c|}
\hline Samples & LTA & Quartz & Kaolinite & Illite & $\mathbf{I} / \mathbf{S}$ & Pyrite & Siderite & Ankerite & Calcite & Rutile & Anorthite & Goyazite & Bassanite \\
\hline TJL12-1 & 8.1 & 61.4 & 30.4 & - & - & 1.7 & 4.2 & 2.3 & - & - & - & - & - \\
\hline TJL12-2 & 14.63 & 66.4 & 32.3 & - & - & 0.4 & 0.4 & 0.5 & - & - & - & - & - \\
\hline TJL12-3 & 8.93 & 65.5 & 30.7 & - & - & 0.6 & 2.7 & 0.5 & - & - & - & - & - \\
\hline TJL12-4 & 1.96 & 56.4 & 22.6 & - & - & 0.6 & 3.8 & 7.3 & 0.7 & - & - & - & - \\
\hline TJL12-5 & 4.77 & 75.1 & 21.1 & - & - & 0.8 & 2 & 1 & - & - & - & - & - \\
\hline TJL12-6 & 4.55 & 28.5 & 20.3 & - & 7.6 & 2.9 & 3.6 & 35 & 2 & - & 8.7 & - & - \\
\hline TJL12-7 & 3.64 & 27.7 & 18.9 & - & 8.1 & 1.6 & 3.9 & 38.2 & 1.6 & - & - & - & - \\
\hline TJL12-8p & 94.29 & 2.6 & 85.2 & - & - & - & 9 & 3.2 & - & - & - & - & - \\
\hline TJL12-9 & 15.82 & 51 & 46.1 & - & - & - & - & 2.8 & - & - & - & - & - \\
\hline TJL12-10 & 9.26 & 57.8 & 30.5 & - & - & - & 3 & 5.2 & - & - & - & 3.5 & - \\
\hline TJL12-11 & 4.08 & 49.4 & 36.5 & - & - & 2.4 & 6 & 3.1 & - & - & - & - & 2.7 \\
\hline TJL12-12 & 12.8 & 26.1 & 41.4 & - & - & 0.6 & 5.2 & 26.6 & - & - & - & - & - \\
\hline TJL12-13 & 7.82 & 27.3 & 9.8 & - & - & 0.9 & 58 & 4.1 & - & - & - & - & - \\
\hline TJL12-14 & 19.3 & 0.5 & - & - & - & - & 79.4 & 6.3 & 13.8 & - & - & - & - \\
\hline TJL12-15f & 93.33 & 24.6 & 43.4 & 24.6 & - & - & 3.3 & 1.4 & - & 2.6 & - & - & - \\
\hline AVE & - & 45.62 & 28.38 & - & 7.85 & 1.25 & 14.35 & 10.22 & 4.53 & - & 8.70 & 3.50 & 2.70 \\
\hline
\end{tabular}




\subsubsection{Oxide Minerals}

Quartz (ranging $0.5 \%$ to $75.1 \%$, mean $45.62 \%$ ) makes up a large percentage of minerals in the coal benches. The content of quartz in the upper benches is higher than that in the lower benches (Figure 5). The quartz in coals is present as infillings in maceral cells and as disseminated particles in macerals and kaolinite matrix. The presence of some angular shaped quartz particles $(10-20 \mu \mathrm{m})$ (Figure 6A,B) indicates that quartz has a detrital origin and was transported over a short distance from the sediment source region which is the Yinshan Upland located to the north of the Datong Coalfield [55,56]. In most cases, the quartz occurs as either fine quartz (less than $5 \mu \mathrm{m}$ ) (Figure 6C-E) disseminating in macerals, or as infillings in maceral cells, indicating an authigenic origin [55,56]. As previously reported, the Yinshan Upland also served as the sediment source region for the Hailiushu Coalfield, Heidaigou Coalfield, and Adaohai Coalfield [57-59]. The content of quartz in the Tongjialiang coals is significantly higher than that in the Heidaigou coals (ranging from bdl-16.4\%, mean 2.5\%), Hailiushu coals (ranging from $0.2-9.6 \%$, mean 1.97\%) and Dantanhao coals (bdl-38.2\%, mean 13.03\%), respectively [57-59].

Under SEM-EDS, rutile was occasionally observed in some coal bench samples (Figure 6F). It occurs as disseminated particles, indicating a syngenetic origin.
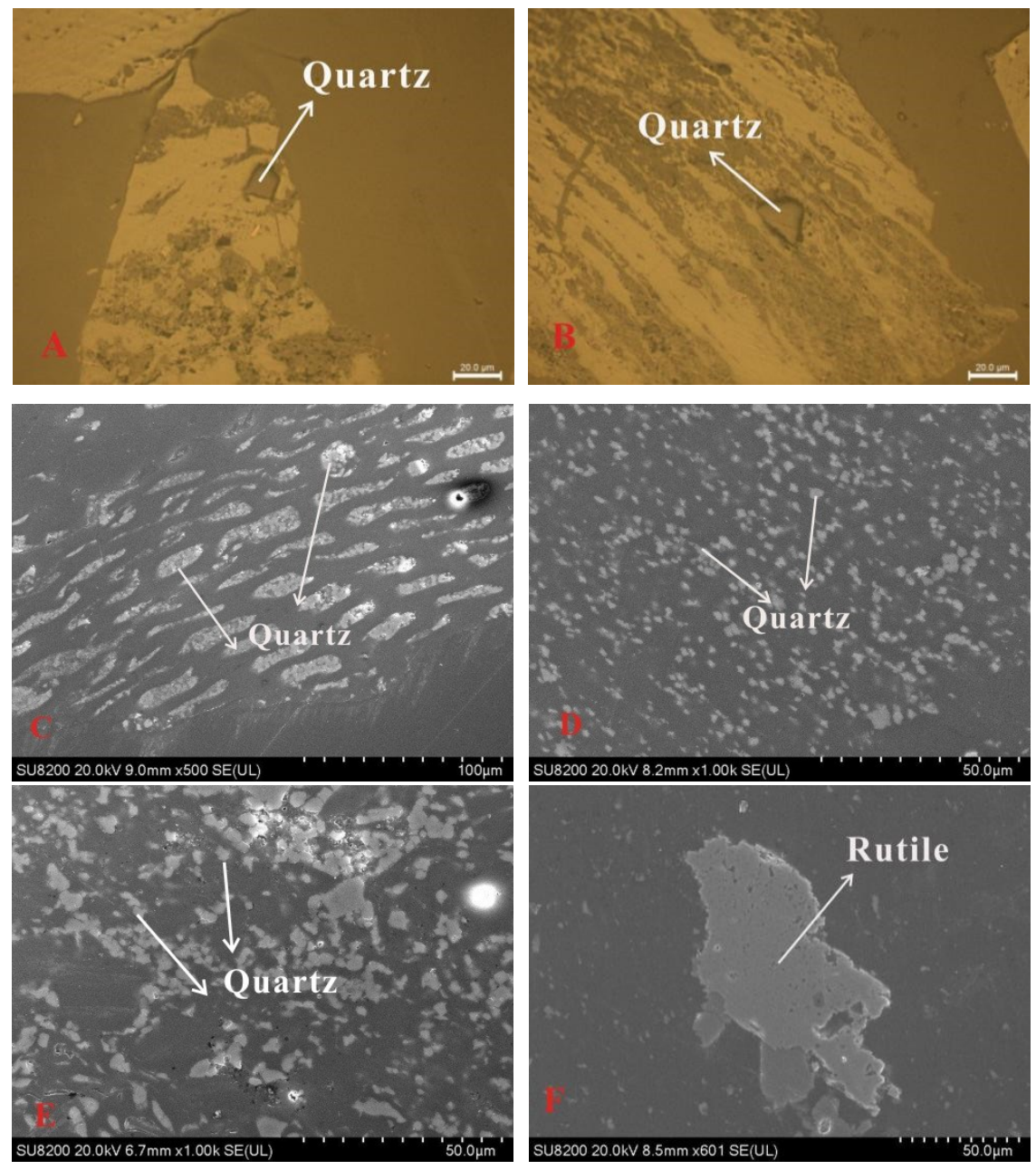

Figure 6. Oxide minerals in the No.12 coal, (A) Quartz disseminated in macerals (reflected light); (B) Quartz disseminated in kaolinite matrix (reflected light); (C) Quartz filled in maceral-cells (SEM, secondary electron image); (D) Quartz disseminated in macerals (SEM, secondary electron image); (E) Quartz disseminated in macerals (SEM, secondary electron image); (F) Rutile disseminated in macerals and kaolinite matrix (SEM, secondary electron image). 


\subsubsection{Clay Minerals}

Kaolinite is another dominant mineral in coal benches, ranging from bdl to $46.1 \%$, with an average of $28.38 \%$. The contents of kaolinite in parting sample (85.2\%) and floor sample (43.4\%) are higher than those in coal benches (Figure 5). Kaolinite mainly occurs as lens-shaped particles (Figure 7A,B) and thin-layers (Figure 7C,D) distributed along the bed plane, indicating detrital materials of terrigenous origin. In addition, a portion of kaolinite filling in maceral cells (Figure 7E,F) indicates formation by authigenic processes [11,12]. In a few cases, kaolinite occurs as infillings in maceral fracture (Figure 7G), suggesting an epigenetic origin [11,12]. The Tongjialiang coals have higher kaolinite content than Heidaigou coals (ranging from 3.6-19.5\%, mean 9\%). However, they have lower kaolinite content than both Hailiushu coals (85-98.5\%, mean 95.6\%) and Datanhao coals (ranging from 13-96.8\%, mean $76.26 \%)$ [57-59].

In addition to the TJL12-15f sample, the proportion of illite in most samples is low, and may be below the detection limit of the XRD and Siroquant techniques. However, illite is also occasionally observed in some samples under SEM. Illite is considered to be formed due to the weathering of feldspar and mica in the sediment source region [11,12]. During the peat accumulation period and the early diagenesis stage, illite was transported to the peat swamp. Illite in No.12 coal disseminates in the maceral and kaolinite matrix, indicating a syngenetic origin (Figure 7I).
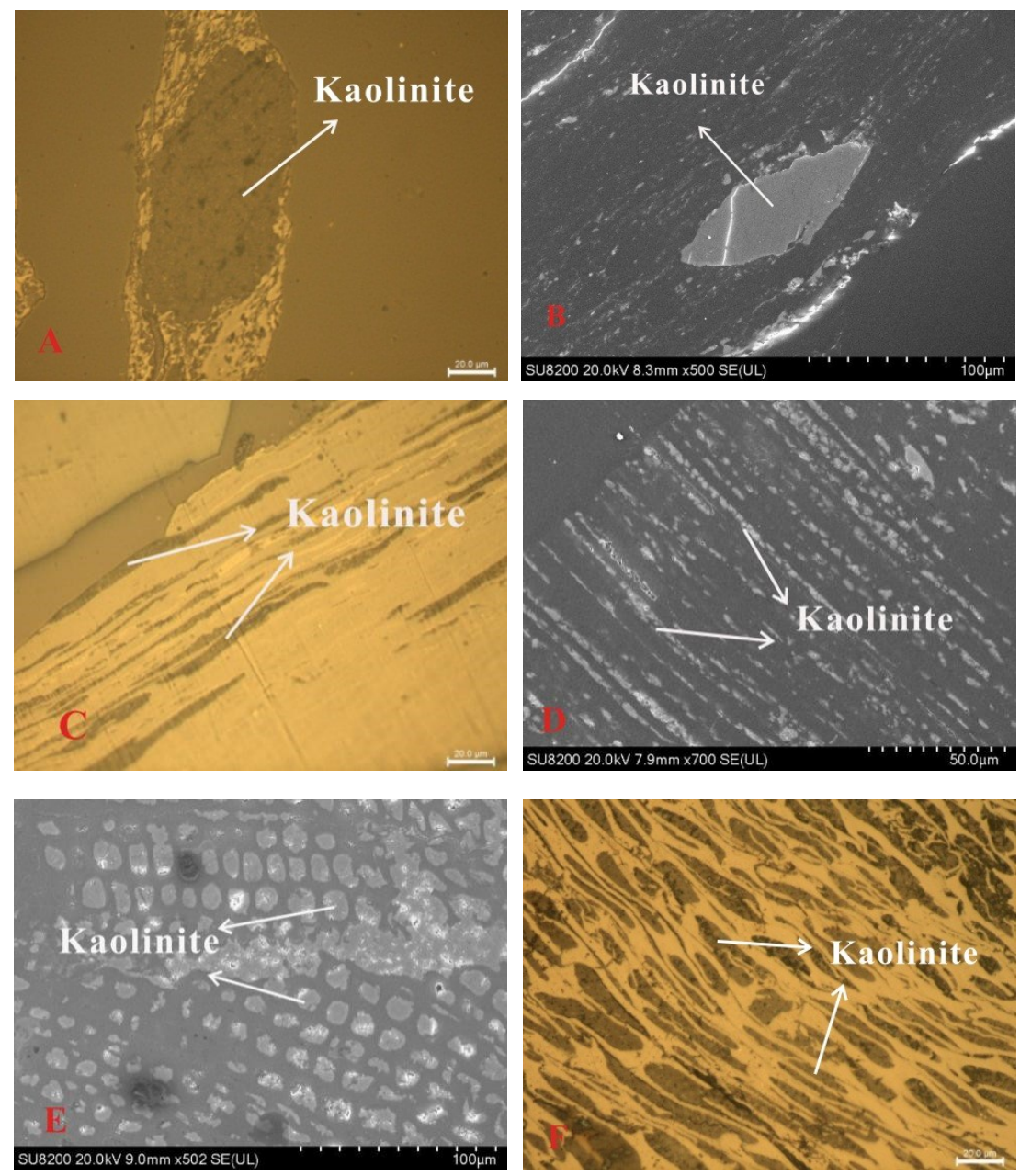

Figure 7. Cont. 

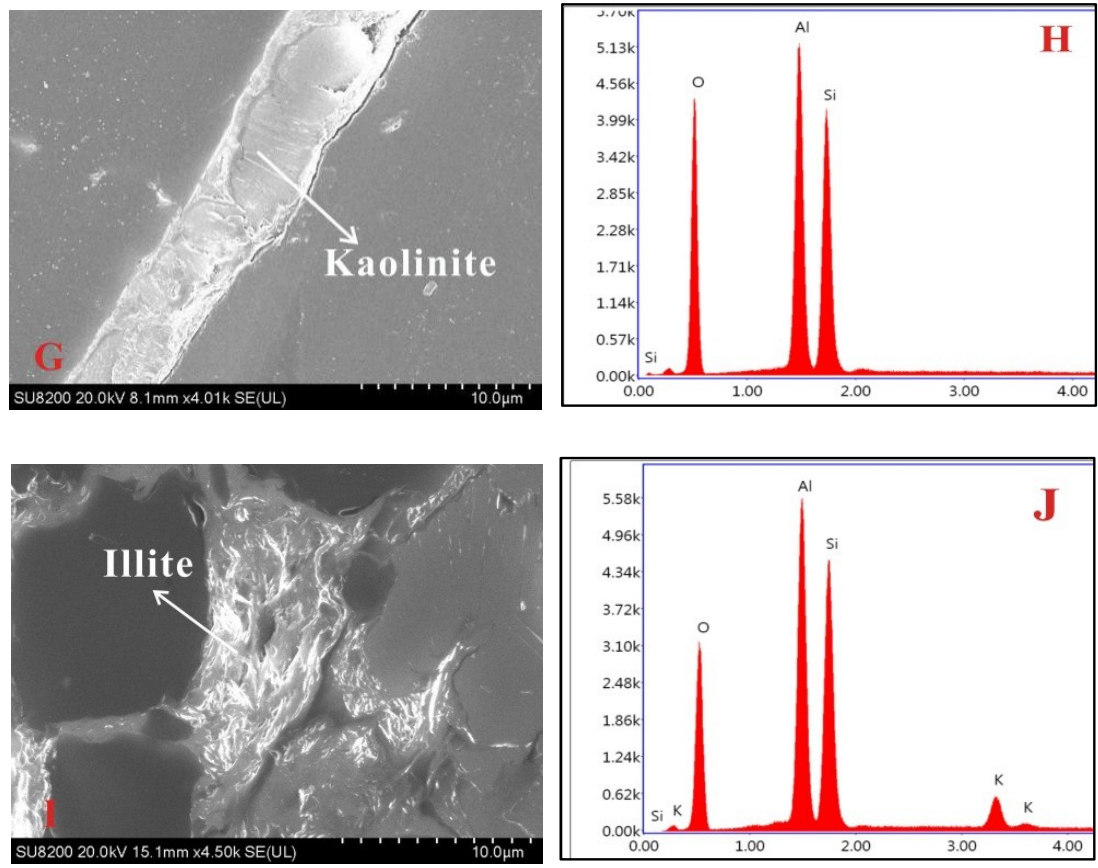

Figure 7. Clay minerals in No.12 coal, (A) lens-shaped kaolinite distributed along the bedding plane (reflected light); (B) lense-shaped kaolinite distributed along the bedding plane (SEM, secondary electron image); (C) thin-layer kaolinite distributed along the bedding plane (reflected light); (D) thin-layer kaolinite distributed along the bedding plane (SEM, secondary electron image); (E) cell-infilling kaolinite in macerals (SEM, secondary electron image); (F) cell-infilling kaolinite in fusinite (reflected light); (G) kaolinite fills in fracture (SEM, secondary electron image); (H) energy dispersive X-ray spectrometry (EDS) of kaolinite; (I) illite (SEM, secondary electron image); (J) energy dispersive X-ray spectrometry (EDS) of illite.

\subsubsection{Sulfide Minerals}

Pyrite is observed and detected in most coal bench samples, and its content ranges from bdl to $2.9 \%$, with an average of $1.25 \%$. Pyrite is distributed as massive forms in the organic matrix (Figure $8 \mathrm{~A}$ ), as disseminated particles in macerals (Figure 8A) and, in a few cases, as infillings (Figure 8B) in maceral-fractures. It can be deduced that pyrite of massive forms and disseminated particles is from a syngenetic origin $[11,12,60,61]$. The fracture-filling pyrite is likely deposited from migrating solutions, suggesting an epigenetic origin [11,12,60,61].
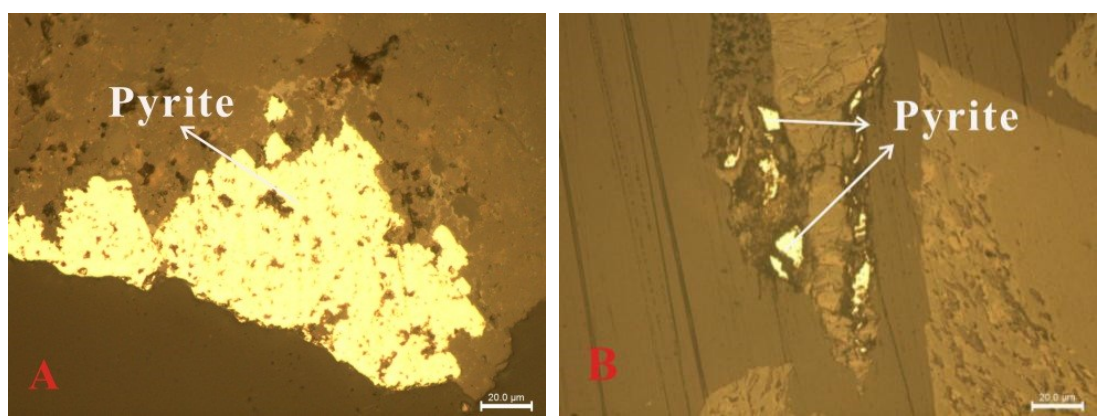

Figure 8. Pyrite in the No.12 coal, (A) pyrite as massive forms distributed in the macerals (reflected light); (B) Pyrite disseminated in macerals (reflected light).

\subsubsection{Carbonate Minerals}

In all the coal bench samples, the content of siderite ranges from bdl to the maximum value of $79.4 \%$ in sample TJL12-14, with an average of $14.35 \%$. The proportion of siderite is significantly 
higher in lower benches (especially in TJL12-13 and TJL12-14) than upper benches (Figure 5). Two modes of occurrence are observed for siderite in coals: coarse-grained massive siderite (Figure 9A) and cell-filling siderite (Figure 9B). The massive siderite is of a syngenetic origin and the cell-filling siderite is probably of an authigenic origin [11,12].
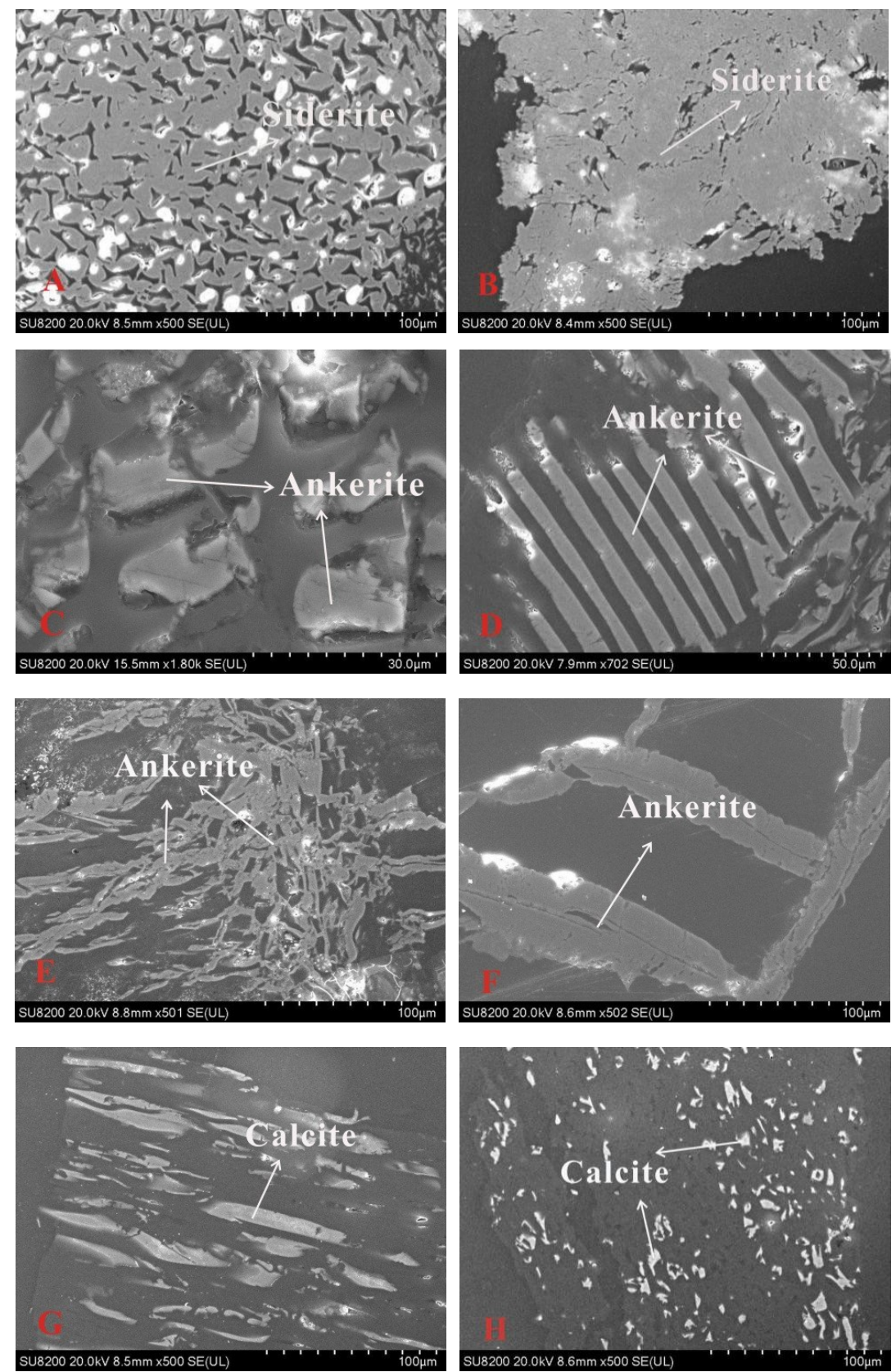

Figure 9. Carbonate minerals in No.12 coal: (A) siderite fills in maceral-cells (SEM, secondary electron image); (B) siderite occurs as coarse-grained massive (SEM, secondary electron image); (C) ankerite fills in maceral-cells (SEM, secondary electron image); (D) ankerite fills in maceral-cells (SEM, secondary electron image); (E) ankerite occurs as veins (SEM, secondary electron image); (F) ankerite fills in maceral-fracture (SEM, secondary electron image); (G) calcite fills in maceral cells (SEM, secondary electron image); (H) calcite fills in maceral cells (SEM, secondary electron image).

Ankerite in the coal LTA residues varies from $0.5 \%$ to $38.2 \%$, with an average of 10.22 . The proportion of ankerite is higher in the LTA of coal samples TJL12-7 and TJL12-9 compared with the other coal benches. SEM-EDS observation shows that ankerite occurred as cell-fillings (Figure 9C,D), 
vein and (Figure 9E) fracture-fillings (Figure 9F). These modes of occurrence indicate that ankerite in the Tongjialiang coals is probably of an epigenetic origin [12,62].

The content of calcite in the coal LTAs is low. Calcite is only detected in samples TJL12-4, TJL12-6, TJL12-7, and TJL12-14 by XRD and Siroquant techniques. SEM-EDS observation shows that calcite occurs as cell-infillings, indicating an authigenic origin (Figure 8G,H) [11,12,62].

\section{Discussion}

\subsection{Sediment Source Region}

The different ratios of $\mathrm{Al}_{2} \mathrm{O}_{3} / \mathrm{TiO}_{2}$ represent different types of sediment source rocks, indicating, in particular, the sediment-source regions for coal deposits [47,48,63]. Ratios of $\mathrm{Al}_{2} \mathrm{O}_{3} / \mathrm{TiO}_{2}$ ranging from 3 to 8 indicate mafic source rocks, 8-21 for intermediate source rocks, and 21-70 for felsic source rocks $[47,48]$. $\mathrm{Al}_{2} \mathrm{O}_{3} / \mathrm{TiO}_{2}$ ratios for the coal bench samples range from 20.63 to 86.62 with an average of 41.20 , indicating that the sediment-source region for the coal and its host rocks is mainly characterized by felsic and intermediate source rocks.

The dominant sediment source of the Jurassic coals from the Datong Coalfield is generally considered to be gneiss and granite of the Yinshan Upland, which is typically enriched in lithophile elements such as $\mathrm{Li}, \mathrm{Be}, \mathrm{F}, \mathrm{Ga}, \mathrm{Zr}, \mathrm{Nb}, \mathrm{Hf}, \mathrm{Ta}, \mathrm{Th}, \mathrm{U}$, etc. [22]. A number of studies have indicated that the coals with sediment source consisting of felsic rocks have high concentration of lithophile elements $[57,64]$. The $\mathrm{Al}_{2} \mathrm{O}_{3} / \mathrm{TiO}_{2}$ ratios indicate that the sediment-source region for the coals and the host rocks is mainly characterized by felsic and intermediate source rocks, corresponding to the characteristics of the gneiss of the Yinshan Upland. In the No.12 coal samples, some lithophile elements, including $\mathrm{Li}, \mathrm{Be}, \mathrm{F}, \mathrm{Zr}, \mathrm{Nb}, \mathrm{Hf}, \mathrm{Ta}$, and $\mathrm{W}$ show slightly higher concentrations. Furthermore, some siderophile elements, including $\mathrm{V}, \mathrm{Cr}, \mathrm{Co}, \mathrm{Ni}, \mathrm{Ge}$, and $\mathrm{Sn}$, are also slightly enriched in the $\mathrm{No} .12$ coal. This may be attributed to the fact that the No.12 coal mainly originated from felsic detrital materials, and to a lesser extent, from the mafic detrital materials.

$\mathrm{Eu}$ anomalies are generally considered to originate from the sediment source region. The ratios of $\mathrm{Al}_{2} \mathrm{O}_{3} / \mathrm{TiO}_{2}$ indicate that the sediment source region of the No.12 coal is characterized by source rocks with felsic and intermediate compositions. Coals with input from felsic and felsic-intermediate detrital materials usually display distinctly negative Eu anomalies [53]. Dai et al. reported that the detrital input of alkali mafic rocks and high-temperature solutions could cause positive Eu anomalies in coals [53]. The L-type enrichment generally reflects a terrigenous origin [51]. The samples of TJL12-4, TJL12-5, TJL12-8p, and TJL12-10, are characterized by L-type REY enrichment patterns, indicating that these samples may originate from the detrital materials of the sediment source region. Furthermore, these samples display no pronounced or weakly negative Eu anomalies. Hence, it can be deduced that these samples may have originated largely from felsic detrital materials and, to a lesser extent, from mafic detrital materials. This may be due to the fact that the mafic detrital materials are characterized by positive $\mathrm{Eu}$ anomalies which would overprint the negative Eu anomalies caused by input of felsic detrital materials [51,53]. The floor sample TJL12-15f is characterized by the L-type REY enrichment pattern and $\mathrm{Al}_{2} \mathrm{O}_{3} / \mathrm{TiO}_{2}$ ratio of 33.06, indicating that the floor sample is probably derived from felsic source rocks. However, this floor sample displays weakly positive Eu anomaly rather than negative $\mathrm{Eu}$ anomaly as expected. The weakly positive Eu anomalies are possibly due to the high content of plagioclase and other feldspars in the granite from the sediment source region resulting from magmatic fractionation or from gravitational separation during the sedimentary processes [65]. Generally, Eu is concentrated in the feldspar of granite, especially in the plagioclase [65]. Therefore, the source rock of the floor sample TJL12-15f is characterized by weakly positive Eu anomalies because a high content of plagioclase was retained in the granite from the sediment source region.

In addition, the formation of syngenetic siderite in No.12 coal is related to the weathering of biotite in the sediment source region [22]. A large proportion of $\mathrm{Fe}^{2+}$ was released by the weathering of biotite in the gneiss of the Yinshan Upland [22]. Subsequently, the Fe-rich water entered into the paleomire where the weakly acidic or weakly alkaline conditions developed. Due to the low sulfur 
content, $\mathrm{Fe}^{2+}$ preferentially combined with $\mathrm{CO}_{2}$ released by organic matter to form a large amount of the syngenetic siderite $[11,12,66]$. Siderite is typically formed when the supply of sulfate ions for bacterial reduction is limited $[11,12,66,67]$. As indicated by Ward, abundant syngenetic siderite usually indicates deposition of coal under non-marine conditions, or under the influence of low sulfate water in the paleomire [11,12].

\subsection{Injection of Low Temperature Hydrothermal Fluids}

The modes of occurrence of the epigenetic ankerite provide further evidence that there may be injection of low temperature hydrothermal fluids into the paleomire $[11,12,62,68]$. As discussed above, ankerite that occurs as veins, cell infillings, and fracture infillings can be considered to be precipitated from Fe-Mg-Ca rich low temperature hydrothermal fluids circulating in the coal seams in the late stage of diagenesis $[11,12,62,69]$. Although some lithophile elements show enrichment, Ta and $\mathrm{W}$ are more enriched than the other lithophile elements in No.12 coal. The correlation coefficients of Ta-ankerite and $\mathrm{W}$-ankerite are 0.76 and 0.75 (Figure 10), respectively, indicating that a portion of Ta and $\mathrm{W}$ in No.12 coal may be derived from the Fe-Mg-Ca rich low temperature hydrothermal fluids.
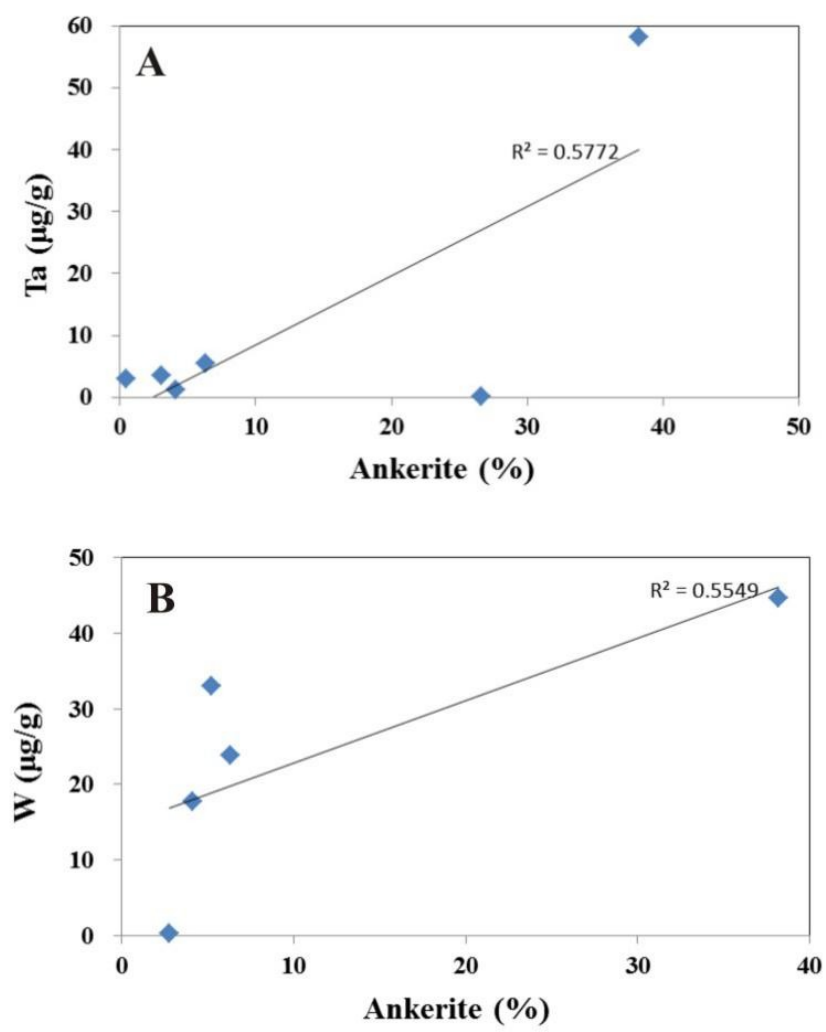

Figure 10. Correlation of: (A) Ta to ankerite and (B) W to ankerite.

Furthermore, it is noteworthy that the siderite is replaced by ankerite in some samples (Figure 11). This is probably because when the hydrothermal fluid invaded into the coal seam in the later diagenesis stage, some epigenetic ankerite was formed as a product of metasomatism between syngenetic siderite and Fe-Mg-Ca rich hydrothermal fluids.

Dai et al. reported that the positive Eu anomaly would be expected to be strongly positive due to the injection of high-temperature hydrothermal solutions $\left(>200^{\circ} \mathrm{C}\right)$ coupled with an extremely reducing environment [53]. Previous investigation by Dai et al. shows that strong positive Eu anomalies in Hongmao Coalfield, Yishan Coalfield, and Luocheng Coalfield from Guangxi Province resulted from the injection of high-temperature hydrothermal solutions $[40,70]$. However, in the present study, the samples with the H-type and $\mathrm{M}-\mathrm{H}$ type enrichment patterns show no pronounced and weakly 
positive Eu anomalies, indicating that the temperature of the hydrothermal fluids circulating in the basin may be relatively low $\left(<200^{\circ} \mathrm{C}\right)[51,53]$.
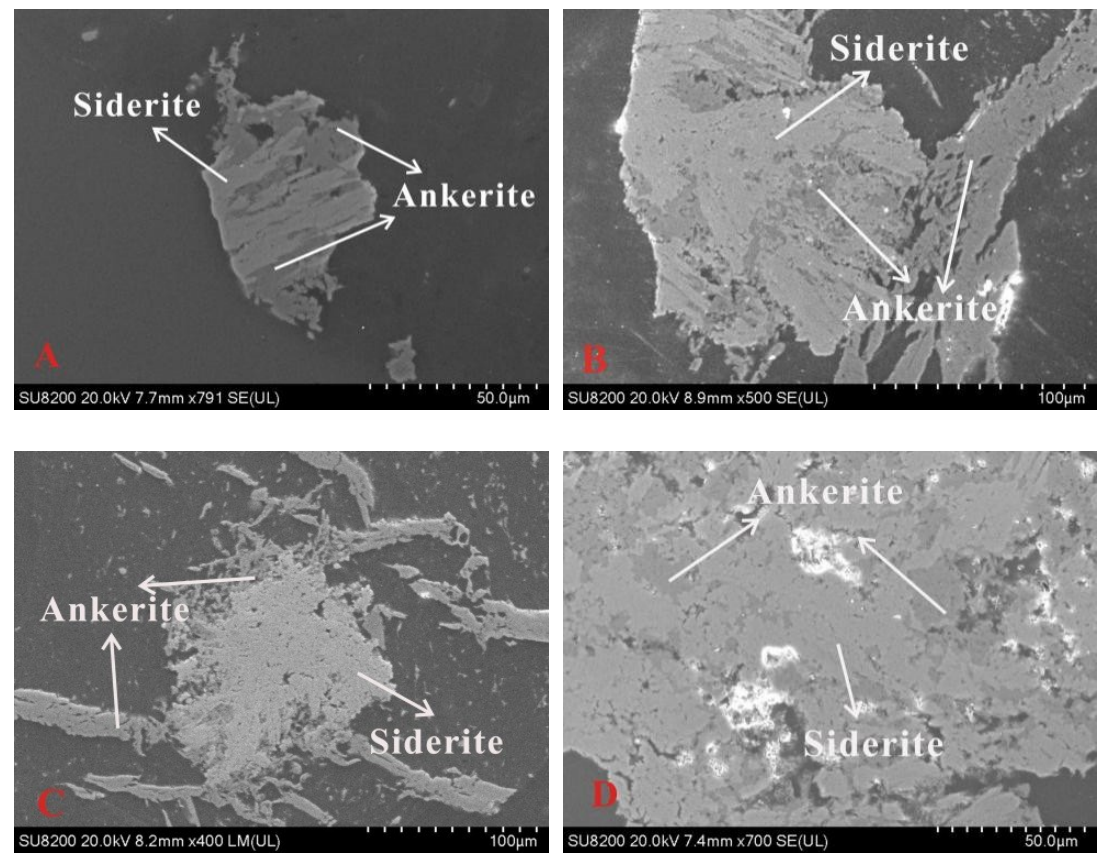

Figure 11. (A-D) Epigenetic ankerite was formed due to the metasomatism between syngenetic siderite and Fe-Mg-Ca rich hydrothermal fluids (SEM, secondary electron image).

The H-type enrichment is probably due to the natural waters enriched in HREY that may circulate in coal basins. The natural waters include marine waters, alkaline terrestrial waters, some cold mineral waters, some low-temperature $\left(130^{\circ} \mathrm{C}\right)$ alkaline hydrothermal solutions, and high-temperature $\left(>500^{\circ} \mathrm{C}\right.$ ) volcanogenic fluids [51]. The formation of Jurassic coals from Datong Coalfield was affected by fresh water [22,69]. Furthermore, the epigenetic ankerite which is derived from the alkaline low temperature hydrothermal fluids is observed in the No.12 coal. No evidence that the HREE enrichment was influenced by the alkaline terrestrial waters, cold mineral waters and high-temperature $\left(>500{ }^{\circ} \mathrm{C}\right)$ volcanogenic fluids is observed in the coals of present study. Therefore, it is believed that the alkaline low temperature hydrothermal fluids may be responsible for the HREE enrichment. This may be due to the fact that the Jurassic coal seams were invaded by the Yanshanian diabase which may be considered as the source of the alkaline low temperature hydrothermal fluids [22]. Additionally, some siderophile elements, such as V, Cr, Co, Ni, Ge, and Sn, show significantly higher concentrations in sample TJL12-1 than other samples. The sample TJL12-1 characterized by weakly positive Eu anomaly and H-type enrichment pattern is probably influenced by the injection of low temperature hydrothermal solutions, which may be the reason for the enrichment of $\mathrm{V}, \mathrm{Cr}, \mathrm{Co}, \mathrm{Ni}, \mathrm{Ge}$, and $\mathrm{Sn}$.

The M-type enrichment is possibly related to acidic natural waters that may circulate in coal basins, including acidic hydrothermal solutions with high REY concentrations [51,71]. Another reason for an M-type enrichment pattern in coals may be the higher sorption of MREY by humic matter in comparison with that of LREY and HREY [51]. As discussed above, the M-H type enrichment is generally attributed to the alkaline low temperature hydrothermal solutions circulating in the basin. It is also ascribed, to a lesser extent, to the acidic hydrothermal fluids and the humic matter in the coals.

\section{Conclusions}

The Middle Jurassic No.12 coal from the Tongjialiang Mine is determined to be a low-medium volatile bituminous coal characterized by low ash yield content, low moisture content, and ultra-low sulfur content. Compared with Chinese coals, the Tongjialiang coals have slightly higher average 
percentages of $\mathrm{MgO}$ and $\mathrm{P}_{2} \mathrm{O}_{5}$, and lower average percentages of the other major oxides, including $\mathrm{SiO}_{2}, \mathrm{TiO}_{2}, \mathrm{Al}_{2} \mathrm{O}_{3}, \mathrm{Fe}_{2} \mathrm{O}_{3}, \mathrm{CaO}, \mathrm{MnO}, \mathrm{Na}_{2} \mathrm{O}$, and $\mathrm{K}_{2} \mathrm{O}$. Compared with the world's hard coals, $\mathrm{Be}$, $\mathrm{Cr}, \mathrm{Co}, \mathrm{Ni}, \mathrm{Ge}, \mathrm{Sn}, \mathrm{Ta}$, and $\mathrm{W}$ are slightly enriched in Tongjialiang coals. The concentrations of $\mathrm{Li}, \mathrm{F}, \mathrm{Sc}, \mathrm{V}, \mathrm{Cu}, \mathrm{Ga}$, Se, Sr, $\mathrm{Zr}, \mathrm{Nb}, \mathrm{Hf}, \mathrm{Pb}$, Th, and $\mathrm{U}$ are close to the average values of the these elements in the worold's hard coals. The minerals in No.12 coal mainly include quartz, kaolinite, siderite, and ankerite, along with a small proportion of pyrite, illite, calcite, and rutile. The modes of occurrence of quartz indicate authigenic and terrigenous origins. Kaolinite has a variety of origins such as authigenic, terrigenous, and epigenetic origins. Siderite, pyrite, calcite, and rutile are probably of syngenetic origins. Ankerite occurs as veins, cell-infillings, and fracture-infillings, indicating an epigenetic origin. The formation of syngenetic siderite in No.12 coal is related to the weathering of biotite in the gneiss of the Yinshan Upland. The modes of occurrence of ankerite indicate that the coals may be affected by the injection of low temperature hydrothermal fluids. Interestingly, a portion of epigenetic ankerite is a product of metasomatism between syngenetic siderite and the epigenetic Fe-Mg-Ca rich hydrothermal fluids.

Based on the ratios of $\mathrm{Al}_{2} \mathrm{O}_{3} / \mathrm{TiO}_{2}$, L-type REY enrichment patterns as well as some enriched lithophile elements and siderophile elements, the sediment source rocks are expected to be mainly from the felsic detrital materials and from the mafic detrital materials to a lesser extent. In addition, the $\mathrm{H}$ and $\mathrm{M}-\mathrm{H}$ type REY enrichment patterns as well as the modes of occurrence of ankerite indicate that the No.12 coal was also influenced by the injection of low temperature hydrothermal fluids into the coal seams.

Author Contributions: Y.Y. designed the experiments and performed both SEM-EDS and XRD. S.T. guided the experiments and analyzed the data. S.Z. analyzed the experimental data. Y.Y. wrote the bulk of this paper. All authors participated in writing this paper.

Funding: This research was funded by the National Key Basic Research Program of China (no. 2014CB238901) and the Key Program of the National Natural Science Foundation of China (no. 41330317).

Acknowledgments: We are particularly grateful for the anonymous reviewers for providing useful suggestions and comments.

Conflicts of Interest: The authors declare no conflict of interest.

\section{References}

1. Swaine, D.J. Trace Elements in Coals; Butterworths: Sydney, Australia, 1990.

2. Ren, D.Y.; Zhao, F.H.; Dai, S.F.; Zhang, J.Y.; Luo, K.L. Geochemistry of Trace Elements in Coal; Science Press: Beijing, China, 2006. (In Chinese with English Abstract)

3. Dai, S.F.; Ren, D.Y.; Chou, C.-L.; Finkelman, R.B.; Seredin, V.V.; Zhou, Y.P. Geochemistry of trace elements in Chinese coals: A review of abundances, genetic types, impacts on human health, and industrial utilization. Int. J. Coal Geol. 2012, 94, 3-21. [CrossRef]

4. Sun, Y.Z.; Zhao, C.L.; Qin, S.J.; Xiao, L.; Li, Z.S.; Lin, M.Y. Occurrence of some valuable elements in the unique "high-aluminium coals" from the Jungar coalfield, China. Ore Geol. Rev. 2016, 72, 659-668. [CrossRef]

5. Dai, S.F.; Yan, X.Y.; Ward, C.R.; Hower, J.C.; Wang, X.B.; Zhao, L.; Ren, D.Y.; Finkelman, R.B. Valuable elements in Chinese coals: A review. Int. Geol. Rev. 2018, 60, 590-620. [CrossRef]

6. Dai, S.F.; Finkelman, R.B. Coal as a promising source of critical elements: Progress and future prospects. Int. J. Coal Geol. 2018, 186, 155-164. [CrossRef]

7. Qin, S.J.; Sun, Y.Z.; Li, Y.H.; Wang, J.X.; Zhao, C.; Gao, K. Coal deposits as promising alternative sources for gallium. Earth Sci. Rev. 2015, 150, 95-101. [CrossRef]

8. Swain, D.J.; Goordarzi, F. Environmental Aspects of Trace Elements in Coal; Energy ampersand Environment volume 2; Kluwer Academic Publishers: Dordrecht, The Netherlands, 1995.

9. Finkelman, R.B.; Tian, L.W. The Health Impacts of Coal Use in China. Int. Geol. Rev. 2018, 60, 579-589. [CrossRef]

10. Tian, H.Z.; Lu, L.; Hao, J.M.; Gao, J.J.; Cheng, K.; Liu, K.Y.; Qin, P.P.; Zhu, C.Y. A review of key hazardous trace elements in Chinese coals: Abundance, occurrence, behavior during coal combustion and their environmental impacts. Energy Fuels 2013, 27, 601-614. [CrossRef]

11. Ward, C.R. Analysis and significance of mineral matter in coal seams. Int. J. Coal Geol. 2002, 50, 135-168. [CrossRef] 
12. Ward, C.R. Analysis, origin and significance of mineral matter in coal: An updated review. Int. J. Coal Geol. 2016, 165, 1-27. [CrossRef]

13. Dai, S.F.; Wang, P.P.; Ward, C.R.; Tang, Y.G.; Song, X.L.; Jiang, J.H.; Hower, J.C.; Li, T.; Seredin, V.V.; Wagner, N.J.; et al. Elemental and mineralogical anomalies in the coal-hosted Ge ore deposit of Lincang, Yunnan, southwestern China: Key role of $\mathrm{N}_{2}-\mathrm{CO}_{2}$ mixed hydrothermal solutions. Int. J. Coal Geol. 2015, 152, 19-46. [CrossRef]

14. Liu, J.J.; Yang, Z.; Yan, X.Y.; Ji, D.; Yang, Y.P.; Hu, L.C. Modes of occurrence of highly-elevated trace elements in superhigh-organic-sulfur coals. Fuel 2015, 156, 190-197. [CrossRef]

15. Zhao, L.; Ward, C.R.; French, D.; Graham, I.T. Mineralogical composition of Late Permian coal seams in the Songzao Coalfield, southwestern China. Int. J. Coal Geol. 2013, 116, 208-226. [CrossRef]

16. Dai, S.F.; Chekryzhov, I.Y.; Seredin, V.V.; Nechaev, V.P.; Graham, I.T.; Hower, J.C.; Ward, C.R.; Ren, D.Y.; Wang, X.B. Metalliferous coal deposits in East Asia (Primorye of Russia and South China): A review of geodynamic controls and styles of mineralization (Review). Gondwana Res. 2016, 29, 60-82. [CrossRef]

17. Qin, Y.; Wang, W.F.; Song, D.Y. Migrating behavior and mechanism of deleterious elements in Taixi coals during cleaning process. J. Fuel Chem. Technol. 2002, 30, 147-150.

18. Spears, D.A. The origin of tonsteins, an overview, and links with seatearths, fireclays and fragmental clay rocks. Int. J. Coal Geol. 2012, 94, 22-31. [CrossRef]

19. Dai, S.F.; Yang, J.Y.; Ward, C.R.; Hower, J.C.; Liu, H.D.; Garrison, T.M.; French, D.; O'Keefe, J.M.K. Geochemical and mineralogical evidence for a coal-hosted uranium deposit in the Yili Basin, Xinjiang, northwestern China. Ore Geol. Rev. 2015, 70, 1-30. [CrossRef]

20. Dou, G.M. Datong Coalfield Carboniferous-Permian Coal Seam Occurrence Characteristics and Control Effects; Taiyuan University of Technology: Taiyuan, China, 2013. (In Chinese with English Abstract)

21. Liu, D.N. The Coupling Relationship of Coal Metamorphism and Sedimentary Tectonic Magmatic Activities for Datong Double Period Coal-Bearing Basin; Taiyuan University of Technology: Taiyuan, China, 2015. (In Chinese with English Abstract)

22. Chen, Y.X.; Dai, D.L. Sedimentary facies of Jurassic in Datong Coalfield in Shanxi Province. Acta Geol. Sin. 1962, 42, 321-332. (In Chinese with English Abstract)

23. Yuan, Y.; Tang, S.H.; Zhang, S.H.; Yang, N. Mineralogical and Geochemical Characteristics of Trace Elements in the Yongdingzhuang Mine, Datong Coalfield, Shanxi Province, China. Minerals 2018, 8, 297. [CrossRef]

24. Wang, J.Y.; Wang, W.F.; Li, J.; Qin, Y. Deposit Features of Ge, Ga and U Elements in Northern Part of Datong Coalfield. Coal Sci. Technol. 2010, 38, 117-121. (In Chinese with English Abstract)

25. Liu, D.N. The Study of Coal Petrology and Coal Geochemistry in Permo-Carboniferous Coals from Datong Coalfield; Taiyuan University of Technology: Taiyuan, China, 2007. (In Chinese with English Abstract)

26. Liu, D.N.; Zhou, A.C.; Chang, Z.G. Geochemistry characteristics of major and rare earth elements in No. 8 raw and weathered coal from Taiyuan Formation of Datong Coalfield. J. China Coal Soc. 2015, 40, 422-430. (In Chinese with English Abstract)

27. Liu, D.N.; Zhou, A.C.; Ma, M.L. Coal facies characteristics of No. 5 coal seam in Baidong Mine Area, Datong Coalfield. Coal Geol. China 2011, 23, 1-4. (In Chinese with English Abstract)

28. ASTM Standard D3177-11. Test Method for Total sulfur in the Analysis Sample of Coal and Coke; ASTM International: West Conshohocken, PA, USA, 2011.

29. ASTM Standard D2492-02. Test Methods for Forms Sulfur in the Analysis Sample of Coal and Coke; ASTM International: West Conshohocken, PA, USA, 2002.

30. ASTM Standard D3173-11. Test Method for Moisture in the Analysis Sample of Coal and Coke; ASTM International: West Conshohocken, PA, USA, 2011.

31. ASTM Standard D3174-11. Test Method for Ash in the Analysis Sample of Coal and Coke; ASTM International: West Conshohocken, PA, USA, 2011.

32. ASTM Standard D3175-11. Test Method for Volatile Matter in the Analysis Sample of Coal and Coke; ASTM International: West Conshohocken, PA, USA, 2011.

33. Taylor, J.C. Computer programs for standardless quantitative analysis of minerals using the full powder diffraction profile. Powder Diffr. 1991, 6, 2-9. [CrossRef]

34. Ward, C.R.; Spears, D.A.; Booth, C.A.; Staton, I.; Gurba, L.W. Mineral matter and trace elements in coals of the Gunnedah Basin, New South Wales, Australia. Int. J. Coal Geol. 1999, 40, 281-308. [CrossRef] 
35. Ward, C.R.; Matulis, C.E.; Taylor, J.C.; Dale, L.S. Quantification of mineral matter in the Argonne Premium coals using interactive Rietveld-based X-ray diffraction. Int. J. Coal Geol. 2001, 46, 67-82. [CrossRef]

36. Ruan, C.D.; Ward, C.R. Quantitative X-ray powder diffraction analysis of clay minerals in Australian coals using Rietveld methods. Appl. Clay Sci. 2002, 21, 227-240. [CrossRef]

37. Dai, S.F.; Wang, X.B.; Zhou, Y.P.; Hower, J.C.; Li, D.H.; Chen, W.M.; Zhu, X.W.; Zou, J.H. Chemical and mineralogical compositions of silicic, mafic, and alkali tonsteins in the Late Permian coals from the Songzao Coalfield, Chongqing, Southwest China. Chem. Geol. 2011, 282, 29-44. [CrossRef]

38. Li, X.; Dai, S.F.; Zhang, W.G.; Li, T.; Zheng, X.; Chen, W.M. Determination of as and Se in coal and coal combustion products using closed vessel microwave digestion and collision/reaction cell technology (CCT) of inductively coupled plasma mass spectrometry (ICP-MS). Int. J. Coal Geol. 2014, 124, 1-4. [CrossRef]

39. ASTM. Standard Test Method for Total Fluorine in Coal and Coke by Pyrohydrolytic Extraction and Ion SelectiveElectrode or Ion Chromatograph Methods; Standard D5987-96, Reapproved 2007; ASTM International: West Conshohocken, PA, USA, 2002.

40. Dai, S.F.; Xie, P.P.; Ward, C.R.; Yan, X.Y.; Guo, W.M.; French, D.; Graham, I.T. Anomalies of rare metals in Lopingian super-high-organic-sulfur coals from the Yishan Coalfield, Guangxi, China. Ore Geol. Rev. 2017, 88, 235-250. [CrossRef]

41. GB/T 15224.1-2010. Classification for Quality of Coal, Part 1; Chinese National Standard; General Administration of Quality Supervision, Inspection and Quarantine of the People's Republic of China: Beijing, China, 2011. (In Chinese)

42. GB/T 15224.2-2010. Classification for Quality of Coal, Part 2; Chinese National Standard; General Administration of Quality Supervision, Inspection and Quarantine of the People's Republic of China: Beijing, China, 2011. (In Chinese)

43. China Coal Research Institute. MT/T849-2000, Classification for Volatile Matter of Coal; Chinese National Standard; General Administration of Quality Supervision, Inspection and Quarantine of the People's Republic of China: Beijing, China, 2000. (In Chinese)

44. China Coal Science Research Institute. MT/T850-2000, Classification for Total Moisture in Coal; Chinese National Standard; General Administration of Quality Supervision, Inspection and Quarantine of the People's Republic of China: Beijing, China, 2000. (In Chinese)

45. ASTM. Standard Classification of Coals by Rank, D388-2015; Reapproved 2007; ASTM International: West Conshohocken, PA, USA, 2015.

46. Chou, C.-L. Sulfur in coals: A review of geochemistry and origins. Int. J. Coal Geol. 2012, 100, 1-13. [CrossRef]

47. Hayashi, K.I.; Fujisawa, H.; Holland, H.D.; Ohmoto, H. Geochemistry of $\sim 1.9$ Ga sedimentary rocks from northeastern Labrador, Canada. Geochim. Cosmochim. Acta 1997, 61, 4115-4137. [CrossRef]

48. He, B.; Xu, Y.G.; Zhong, Y.T.; Guan, J.P. The Guadalupian-Lopingian boundary mudstones at Chaotian (SW China) are clastic rocks rather than acidic tuffs: Implication for a temporal coincidence between the end-Guadalupian mass extinction and the Emeishan volcanism. Lithos 2010, 119, 10-19. [CrossRef]

49. Dai, S.F.; Seredin, V.V.; Ward, C.R.; Hower, J.C.; Xing, Y.W.; Zhang, W.G.; Song, W.J.; Wang, P.P. Enrichment of U-Se-Mo-Re-V in coals preserved within marine carbonate successions: Geochemical and mineralogical data from the Late Permian Guiding Coalfield, Guizhou, China. Miner. Depos. 2015, 50, 159-186. [CrossRef]

50. Ketris, M.P.; Yudovich, Y.E. Estimations of clarkes for carbonaceous biolithes: World average for trace element concentrations in black shales and coals. Int. J. Coal Geol. 2009, 78, 135-148. [CrossRef]

51. Seredin, V.V.; Dai, S.F. Coal deposits as potential alternative sources for lanthanides and yttrium. Int. J. Coal Geol. 2012, 94, 67-93. [CrossRef]

52. Taylor, S.R.; McLennan, S.M. The Continental Crust: Its Composition and Evolution; Blackwell: London, UK, 1985; p. 312.

53. Dai, S.F.; Graham, I.T.; Ward, C.R. A review of anomalous rare earth elements and yttrium in coal. Int. J. Coal Geol. 2016, 159, 82-95. [CrossRef]

54. Yan, X.Y.; Dai, S.F.; Graham, I.T.; He, X.; Shan, K.H.; Liu, X. Determination of Eu concentrations in coal, fly ash and sedimentary rocks using a cation exchange resin and inductively coupled plasma mass spectrometry (ICP-MS). Int. J. Coal Geol. 2018, 19, 152-156. [CrossRef]

55. Sykes, R.; Lindqvist, J.K. Diagenetic quartz and amorphous silica in New Zealand coals. Org. Geochem. 1993, 20, 855-866. [CrossRef] 
56. Dai, S.F.; Tian, L.W.; Chou, C.-L.; Zhou, Y.P.; Zhang, M.Q.; Zhao, L.; Wang, J.M.; Yang, Z.; Cao, H.Z.; Ren, D.Y. Mineralogical and compositional characteristics of Late Permian coals from an area of high lung cancer rate in Xuanwei, Yunnan, China: Occurrence and origin of quartz and chamosite. Int. J. Coal Geol. 2008, 76, 318-327. [CrossRef]

57. Dai, S.F.; Li, T.J.; Jiang, Y.F.; Ward, C.R.; Hower, J.C.; Sun, J.H.; Liu, J.J.; Song, H.J.; Wei, J.; Li, Q.Q.; et al. Mineralogical and geochemical compositions of the Pennsylvanian coal in the Hailiushu Mine, Daqingshan Coalfield, Inner Mongolia, China: Implications of sediment-source region and acid hydrothermal solutions. Int. J. Coal Geol. 2015, 137, 92-110. [CrossRef]

58. Dai, S.F.; Ren, D.Y.; Chou, C.-L.; Li, S.S.; Jiang, Y.F. Mineralogy and geochemistry of the No.6 Coal (Pennsylvanian) in the Junger Coalfield, Ordos Basin, China. Int. J. Coal Geol. 2006, 66, 253-270. [CrossRef]

59. Zhao, L.; Sun, J.H.; Guo, W.M.; Wang, P.P.; Ji, D.P. Mineralogy of the Pennsylvanian coal seam in the Datanhao mine, Daqingshan Coalfield, Inner Mongolia, China: Genetic implications for mineral matter in coal deposited in an intermontane basin. Int. J. Coal Geol. 2016, 167, 201-214. [CrossRef]

60. Dai, S.F.; Li, T.; Seredin, V.V.; Ward, C.R.; Hower, J.C.; Zhou, Y.P.; Zhang, M.Q.; Song, X.; Song, W.J.; Zhao, C.L. Origin of minerals and elements in the Late Permian coals, tonsteins, and host rocks of the Xinde Mine, Xuanwei, eastern Yunnan, China. Int. J. Coal Geol. 2014, 121, 53-78. [CrossRef]

61. Querol, X.; Chinenon, S.; Lopez-Soler, A. Iron sulfide precipitation sequence in Albian coals from the Maestrazgo basin, southeastern Iberean range, northeastern Spain. Int. J. Coal Geol. 1989, 11, 171-189. [CrossRef]

62. Dai, S.F.; Chou, C.-L.; Yue, M.; Luo, K.L.; Ren, D.Y. Mineralogy and geochemistry of a Late Permian coal in the Dafang Coalfield, Guizhou, China: Influence from siliceous and iron-rich calcic hydrothermal fluids. Int. J. Coal Geol. 2005, 61, 241-258. [CrossRef]

63. Dai, S.F.; Liu, J.J.; Ward, C.R.; Hower, J.C.; French, D.; Jia, S.H.; Hood, M.M.; Garrison, T.M. Mineralogical and geochemical compositions of Late Permian coals and host rocks from the Guxu Coalfield, Sichuan Province, China, with emphasis on enrichment of rare metals. Int. J. Coal Geol. 2016, 166, 71-95. [CrossRef]

64. Dai, S.F.; Hower, J.C.; Ward, C.R.; Guo, W.M.; Song, H.J.; O’Keefe, J.M.K.; Xie, P.P.; Hood, M.M.; Yan, X.Y. Elements and phosphorus minerals in the middle Jurassic inertinite-rich coals of the Muli Coalfield on the Tibetan Plateau. Int. J. Coal Geol. 2015, 144, 23-47. [CrossRef]

65. Dai, S.; Guo, W.; Nechaev, V.P.; French, D.; Ward, C.R.; Spiro, B.F.; Finkelman, R.B. Modes of occurrence and origin of mineral matter in the Palaeogene coal (No.19-2) from the Hunchun Coalfield, Jilin Province, China. Int. J. Coal Geol. 2018, 189, 94-110. [CrossRef]

66. Hao, L.S. The origination and geological significance of siderite in Jurassic seams in Datong. Shanxi Ming Inst. Learn. J. 1988, 6, 413-422. (In Chinese with English Abstract)

67. Gould, K.W.; Smith, J.W. The genesis and isotopic composition of carbonates associated with some Permian Australian coals. Chem. Geol. 1979, 24, 137-150. [CrossRef]

68. Karayiğit, A.İ.; Littke, R.; Querol, X.; Jones, T.; Oskay, R.G.; Christanis, K. The Miocene coal seams in the Soma Basin (W. Turkey): Insights from coal petrography, mineralogy and geochemistry. Int. J. Coal Geol. 2017, 173, 110-128. [CrossRef]

69. Wang, X.B.; Dai, S.F.; Chou, C.-L.; Zhang, M.Q.; Wang, J.; Song, X.L.; Wang, W.; Jiang, Y.F.; Zhou, Y.P.; Ren, D.Y. Mineralogy and geochemistry of Late Permian coals from the Taoshuping Mine, Yunnan Province, China: Evidences for the sources of minerals. Int. J. Coal Geol. 2012, 96-97, 49-59. [CrossRef]

70. Dai, S.F.; Ji, D.P.; Ward, C.R.; French, D.; Hower, J.C.; Yan, X.Y.; Wei, Q. Mississippian anthracites in Guangxi Province, southern China: Petrological, mineralogical, and rare earth element evidence for high-temperature solutions. Int. J. Coal Geol. 2018, 197, 84-114. [CrossRef]

71. Johanneson, K.H.; Zhou, X. Geochemistry of the rare earth element in natural terrestrial waters: A review of what is currently known. Chin. J. Geochem. 1997, 16, 20-42. [CrossRef]

(C) 2019 by the authors. Licensee MDPI, Basel, Switzerland. This article is an open access article distributed under the terms and conditions of the Creative Commons Attribution (CC BY) license (http://creativecommons.org/licenses/by/4.0/). 